DOI: http://dx.doi.org/10.21123/bsj.2018.15.4.0455

\title{
On Free Resolution of Weyl Module and Zero Characteristic Resolution In The Case of Partition $(8,7,3)$
}

\author{
Haytham Razooki Hassan ${ }^{1^{*}}$ \\ Niran Sabah Jasim ${ }^{2}$ \\ Received 15/4 /2018, Accepted 2/10/2018, Published 9/12/2018 \\ This work is licensed under a Creative Commons Attribution 4.0 International License.
}

\begin{abstract}
:
This treatise is an application of the characteristic-free resolution of $K_{(8,7,3)} \mathcal{F}$ to the Lascoux resolution of $K_{(8,7,3)} \mathcal{F}$ (characteristic zero resolution). From this, study, we gain the connection between the resolution of Weyl module $\mathrm{K}_{(8,7,3)} \mathcal{F}$ in characteristic free mode and in the Lascoux mode.
\end{abstract}

Keywords: Weyl module, resolution, free resolution, characteristic-free resolution, characteristic zero resolution.

\section{Introduction:}

Let $\mathcal{R}$ be a commutative ring with 1 and $\mathcal{F}$ be a free $\mathcal{R}$-module. The divided power algebra $\mathcal{D F}=\sum_{i \geq 0} \mathcal{D}_{i} \mathcal{F}$ can be acquainted as the graded abelian algebra generated by $x^{i}$ where $x \in \mathcal{F}$ and $i$ is a non-negative integer, and $\mathcal{D}_{i} \mathcal{F}$ is the divided power algebra of degree $i$.

The resolution of partition $\left(p+t_{1}+t_{2}, q+\right.$ $\left.t_{2}, r\right)$ which is represented by below diagram and in our case $t_{1}=t_{2}=0$.

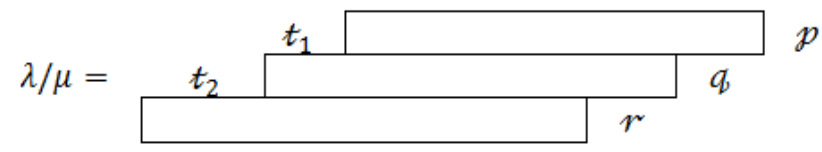

The authors in (1) and (2) clarify the description of the characteristic zero skeleton by Lascoux in the resolution of skew-shapes. While the authors in (3), (4) and (5) exhibit the formulation of the terms of Lascoux resolution.

The authors in (6) exhibit the terms and the exactness of the Weyl resolution in the case of partition $(8,7)$. As well in (7) they discuss the terms of characteristic zero complex in the case of the partition $(8,7,3)$ and the diagram for the complex of characteristic zero in the case of the partition $(8,7,3)$.

In the next section, we survey the terms of characteristic free resolution of Weyl module in the case of the partition $(8,7,3)$ which is the generalization of the partition $(3,3,3)$.

1 Department of Mathematics, College of Science, Mustansiriyah University, Baghdad, Iraq.

2 Department of Mathematics, College of Education for Pure Science/ Ibn Al-Haitham, University of Baghdad, Baghdad, Iraq.

*Corresponding author: haythamhassaan@yahoo.com
While in the last section we stratify the resolution gain it in the below section to the Lascoux resolution by itself track of authors in (8) and (9) with Capelli identities as in (10).

\section{Characteristic-Free Resolution of the Partition $(8,7,3)$}

As in (3) for the case $(p, q, r)$ with $r \geq 2$ the terms of the resolution are:

$$
\begin{aligned}
& \operatorname{Res}([p, q ; 0]) \otimes \mathcal{D}_{r} \oplus \sum_{e \geq 0} \underline{Z}_{32}^{(e+1)} y \operatorname{Res}([p, q+ \\
& e+1 ; e+1]) \otimes \mathcal{D}_{r-e-1} \oplus \\
& \sum_{e_{1} \geq 0, e_{2} \geq e_{1}} \underline{Z}_{32}^{\left(e_{2}+1\right)} y \underline{Z}_{31}^{\left(e_{1}+1\right)} z \operatorname{Res}\left(\left[p+e_{1}+\right.\right. \\
& \left.\left.1, q+e_{2}+1 ; e_{2}-e_{1}\right]\right) \otimes \mathcal{D}_{r-\left(e_{1}+e_{2}+2\right)}
\end{aligned}
$$

where $\underline{Z}_{a b}^{(m)}$ is the following bar complex

$0 \rightarrow \underbrace{Z_{a b} w Z_{a b} w \ldots Z_{a b}}_{m \text {-times }}$

$\longrightarrow \sum_{k_{i} \geq 1, \sum k_{i}=m} z_{a b}^{\left(k_{1}\right)} w Z_{a b}^{\left(k_{2}\right)} w \ldots z_{a b}^{\left(k_{m-1}\right)} \rightarrow$

$\cdots \rightarrow Z_{a b}^{(m)} \rightarrow 0$

By stratify the above formulation for partition $(8,7,3)$

$\operatorname{Res}([8,7 ; 0]) \otimes \mathcal{D}_{3} \oplus \sum_{e \geq 0} \underline{Z}_{32}^{(e+1)}$ y $\operatorname{Res}([8,7+e+$ $1 ; e+1]) \otimes \mathcal{D}_{3-e-1} \oplus$

$\sum_{e_{1} \geq 0, e_{2} \geq e_{1}} \underline{Z}_{32}^{\left(e_{2}+1\right)} y \underline{Z}_{31}^{\left(e_{1}+1\right)} z \operatorname{Res}\left(\left[8+e_{1}+1,7+\right.\right.$ $\left.\left.e_{2}+1 ; e_{2}-e_{1}\right]\right) \otimes \mathcal{D}_{3-\left(e_{1}+e_{2}+2\right)}$

... 1

So

$\sum_{e \geq 0} \underline{Z}_{32}^{(e+1)} y \operatorname{Res}([8,7+e+1 ; e+1])$

$\otimes \mathcal{D}_{3-e-1}=$

$\underline{Z}_{32} y \operatorname{Res}([8,8 ; 1]) \otimes \mathcal{D}_{2} \oplus \underline{Z}_{32}^{(2)} y \operatorname{Res}([8,9 ; 2]) \otimes \mathcal{D}_{1}$

$\oplus \underline{Z}_{32}^{(3)} y \operatorname{Res}([8,10 ; 3]) \otimes \mathcal{D}_{0}$

And 
$\sum_{e_{1} \geq 0, e_{2} \geq e_{1}} \underline{z}_{32}^{\left(e_{2}+1\right)} y \underline{z}_{31}^{\left(e_{1}+1\right)} z$

$\operatorname{Res}\left(\left[8+e_{1}+1,7+e_{2}+1 ; e_{2}-e_{1}\right]\right) \otimes$

$\mathcal{D}_{3-\left(e_{1}+e_{2}+2\right)}=$

$\underline{Z}_{32} y \underline{Z}_{31} z \operatorname{Res}([9,8 ; 0]) \otimes \mathcal{D}_{1} \oplus \underline{Z}_{32}^{(2)} y \underline{Z}_{31} z$

$\operatorname{Res}([9,9 ; 1]) \otimes \mathcal{D}_{0}$

Where $\underline{Z}_{32} y$ is the bar complex

$0 \rightarrow z_{32} y \stackrel{\partial_{y}}{\longrightarrow} Z_{32} \rightarrow 0$

$\underline{z}_{32}^{(2)} y$ is the bar complex

$0 \rightarrow z_{32} y z_{32} y \stackrel{\partial_{y}}{\longrightarrow} z_{32}^{(2)} y \stackrel{\partial_{y}}{\longrightarrow} z_{32}^{(2)} \rightarrow 0$

$\underline{z}_{32}^{(3)} y$ is the bar complex

$$
\begin{gathered}
0 \rightarrow z_{32} y z_{32} y z_{32} y \stackrel{\partial_{y}}{\longrightarrow} \underset{\partial_{y}}{z_{32} y z_{32}^{(2)} y} Z_{32}^{(3)} y \stackrel{\partial_{y}}{\longrightarrow} Z_{32}^{(3)} \rightarrow 0
\end{gathered}
$$

and $\underline{Z}_{31} z$ is the bar complex

$0 \rightarrow Z_{31} z \stackrel{\partial_{z}}{\longrightarrow} Z_{31} \rightarrow 0$

Where $x, y$ and $z$ stand for the separator variables, and the boundary map is $\partial_{x}+\partial_{y}+\partial_{z}$.

Let $\operatorname{Bar}(M, A ; S)$ be the free bar module on the set $S=\{x, y, z\}$, where $A$ is the free associative algebra generated by $Z_{21}, Z_{32}$ and $Z_{31}$ and their divided powers with the pursue relations:

$z_{32}^{(a)} z_{31}^{(b)}=Z_{31}^{(b)} z_{32}^{(a)} \quad$ and $\quad z_{21}^{(a)} z_{31}^{(b)}=z_{31}^{(b)} z_{21}^{(a)}$ and the module $M$ is the direct sum of $\mathcal{D}_{p} \otimes \mathcal{D}_{q} \otimes$ $\mathcal{D}_{r}$ for suitable $p, q$ and $r$ with the action of $Z_{21}, Z_{32}$, and $Z_{31}$ and their divided powers.

The terms of the characteristic-free resolution 1 are:

○ In dimension zero $\left(\chi_{0}\right)$ we have $\mathcal{D}_{8} \otimes \mathcal{D}_{7} \otimes \mathcal{D}_{3}$

- In dimension one $\left(X_{1}\right)$ we have

- $z_{21}^{(b)} x \mathcal{D}_{8+b} \otimes \mathcal{D}_{7-b} \otimes \mathcal{D}_{3} ;$ with $1 \leq b \leq 7$, $b \in \mathbb{Z}^{+}$

- $z_{32}^{(b)} y \mathcal{D}_{8} \otimes \mathcal{D}_{7+b} \otimes \mathcal{D}_{3-b} ;$ with $1 \leq b \leq 3$, $b \in \mathbb{Z}^{+}$

- In dimension two $\left(X_{2}\right)$ we have the sum of the following terms:

- $Z_{21}^{\left(b_{1}\right)} x Z_{21}^{\left(b_{2}\right)} x \mathcal{D}_{8+|b|} \otimes \mathcal{D}_{7-|b|} \otimes \mathcal{D}_{3} ;$ with $2 \leq|b|=b_{1}+b_{2} \leq 7 ; b_{1}, b_{2} \in \mathbb{Z}^{+}$

- $Z_{32} y Z_{21}^{(b)} x \mathcal{D}_{8+b} \otimes \mathcal{D}_{8-b} \otimes \mathcal{D}_{2} ;$ with $2 \leq b \leq 8, b \in \mathbb{Z}^{+}$

- $Z_{32}^{(2)} y Z_{21}^{(b)} x \mathcal{D}_{8+b} \otimes \mathcal{D}_{9-b} \otimes \mathcal{D}_{1} ;$ with $3 \leq b \leq 9, b \in \mathbb{Z}^{+}$

- $z_{32}^{(3)} y Z_{21}^{(b)} x \mathcal{D}_{8+b} \otimes \mathcal{D}_{10-b} \otimes \mathcal{D}_{0} ;$ with $4 \leq b \leq 10, b \in \mathbb{Z}^{+}$

- $z_{32}^{\left(b_{1}\right)} y z_{32}^{\left(b_{2}\right)} y \mathcal{D}_{8} \otimes \mathcal{D}_{7+|b|} \otimes \mathcal{D}_{3-|b|} ;$ with $2 \leq|b|=b_{1}+b_{2} \leq 3 ; b_{1}, b_{2} \in \mathbb{Z}^{+}$

- $Z_{32}^{(b)} y Z_{31} z \mathcal{D}_{9} \otimes \mathcal{D}_{7+b} \otimes \mathcal{D}_{2-b} ;$ with $1 \leq b \leq 2$, $b \in \mathbb{Z}^{+}$
- In dimension three $\left(x_{3}\right)$ we have the sum of the following terms:

- $Z_{21}^{\left(b_{1}\right)} x Z_{21}^{\left(b_{2}\right)} x Z_{21}^{\left(b_{3}\right)} x \mathcal{D}_{8+|b|} \otimes \mathcal{D}_{7-|b|} \otimes \mathcal{D}_{3} ;$ with $3 \leq|b|=b_{1}+b_{2}+b_{3} \leq 7$ and $b_{1} \geq 1$; $b_{1}, b_{2}, b_{3} \in \mathbb{Z}^{+}$

- $Z_{32} y Z_{21}^{\left(b_{1}\right)} x Z_{21}^{\left(b_{2}\right)} x \mathcal{D}_{8+|b|} \otimes \mathcal{D}_{8-|b|} \otimes \mathcal{D}_{2}$; with $3 \leq|b|=b_{1}+b_{2} \leq 8$ and $b_{1} \geq 2 ; b_{1}, b_{2} \in \mathbb{Z}^{+}$

- $z_{32}^{(2)} y z_{21}^{\left(b_{1}\right)} x z_{21}^{\left(b_{2}\right)} x \mathcal{D}_{8+|b|} \otimes \mathcal{D}_{9-|b|} \otimes \mathcal{D}_{1}$; with $4 \leq|b|=b_{1}+b_{2} \leq 9$ and $b_{1} \geq 3 ; b_{1}, b_{2} \in \mathbb{Z}^{+}$

- $z_{32} y Z_{32} y Z_{21}^{(b)} x \mathcal{D}_{8+b} \otimes \mathcal{D}_{9-b} \otimes \mathcal{D}_{1}$; with $3 \leq b \leq 9, b \in \mathbb{Z}^{+}$

- $z_{32}^{(3)} y z_{21}^{\left(b_{1}\right)} x z_{21}^{\left(b_{2}\right)} x \mathcal{D}_{8+|b|} \otimes \mathcal{D}_{10-|b|} \otimes \mathcal{D}_{0} ;$ with $5 \leq|b|=b_{1}+b_{2} \leq 10$ and $b_{1} \geq 4 ; b_{1}, b_{2} \in \mathbb{Z}^{+}$

- $z_{32}^{\left(c_{1}\right)} y z_{32}^{\left(c_{2}\right)} y z_{21}^{(b)} x \mathcal{D}_{8+b} \otimes \mathcal{D}_{10-b} \otimes \mathcal{D}_{0}$; with $c_{1}+c_{2}=3$ and $4 \leq b \leq 10 ; b, c_{1}, c_{2} \in \mathbb{Z}^{+}$

- $Z_{32} y Z_{32} y Z_{32} y \mathcal{D}_{8} \otimes \mathcal{D}_{10} \otimes \mathcal{D}_{0}$

- $z_{32} y z_{31} z Z_{21}^{(b)} x \mathcal{D}_{9+b} \otimes \mathcal{D}_{8-b} \otimes \mathcal{D}_{1} ;$ with $1 \leq b \leq 8, b \in \mathbb{Z}^{+}$

- $z_{32}^{(2)} y Z_{31} z Z_{21}^{(b)} x \mathcal{D}_{9+b} \otimes \mathcal{D}_{9-b} \otimes \mathcal{D}_{0} ;$ with $2 \leq b \leq 9, b \in \mathbb{Z}^{+}$

- $Z_{32} y Z_{32} y Z_{31} z \mathcal{D}_{9} \otimes \mathcal{D}_{9} \otimes \mathcal{D}_{0}$

- In dimension four $\left(X_{4}\right)$ we have the sum of the following terms:

- $Z_{21}^{\left(b_{1}\right)} x Z_{21}^{\left(b_{2}\right)} x Z_{21}^{\left(b_{3}\right)} x z_{21}^{\left(b_{4}\right)} x \mathcal{D}_{8+|b|} \otimes \mathcal{D}_{7-|b|} \otimes \mathcal{D}_{3}$; with $4 \leq|b|=\sum_{i=1}^{4} b_{i} \leq 7$ and $b_{1} \geq 1$; $b_{1}, b_{2}, b_{3}, b_{4} \in \mathbb{Z}^{+}$

- $Z_{32} y Z_{21}^{\left(b_{1}\right)} x Z_{21}^{\left(b_{2}\right)} x Z_{21}^{\left(b_{3}\right)} x \mathcal{D}_{8+|b|} \otimes \mathcal{D}_{8-|b|} \otimes \mathcal{D}_{2}$; with $4 \leq|b|=b_{1}+b_{2}+b_{3} \leq 8$ and $b_{1} \geq 2$; $b_{1}, b_{2}, b_{3} \in \mathbb{Z}^{+}$

- $Z_{32}^{(2)} y Z_{21}^{\left(b_{1}\right)} x Z_{21}^{\left(b_{2}\right)} x Z_{21}^{\left(b_{3}\right)} x \mathcal{D}_{8+|b|} \otimes \mathcal{D}_{9-|b|} \otimes \mathcal{D}_{1}$; with $5 \leq|b|=b_{1}+b_{2}+b_{3} \leq 9$ and $b_{1} \geq 3$; $b_{1}, b_{2}, b_{3} \in \mathbb{Z}^{+}$

- $Z_{32} y Z_{32} y Z_{21}^{\left(b_{1}\right)} x Z_{21}^{\left(b_{2}\right)} x \mathcal{D}_{8+|b|} \otimes \mathcal{D}_{9-|b|} \otimes \mathcal{D}_{1}$; with $4 \leq|b|=b_{1}+b_{2} \leq 9$ and $b_{1} \geq 3$; $b_{1}, b_{2} \in \mathbb{Z}^{+}$

- $Z_{32}^{(3)} y Z_{21}^{\left(b_{1}\right)} x Z_{21}^{\left(b_{2}\right)} x Z_{21}^{\left(b_{3}\right)} x \mathcal{D}_{8+|b|} \otimes \mathcal{D}_{10-|b|} \otimes \mathcal{D}_{0}$; with $6 \leq|b|=b_{1}+b_{2}+b_{3} \leq 10$ and $b_{1} \geq 4$; $b_{1}, b_{2}, b_{3} \in \mathbb{Z}^{+}$

- $Z_{32}^{\left(c_{1}\right)} y Z_{32}^{\left(c_{2}\right)} y Z_{21}^{\left(b_{1}\right)} x Z_{21}^{\left(b_{2}\right)} x \mathcal{D}_{8+|b|} \otimes \mathcal{D}_{10-|b|} \otimes \mathcal{D}_{0}$; with $c_{1}+c_{2}=3$ and $5 \leq|b|=b_{1}+b_{2} \leq 10$ and $b_{1} \geq 4 ; b_{1}, b_{2}, c_{1}, c_{2} \in \mathbb{Z}^{+}$

- $Z_{32} y Z_{32} y Z_{32} y Z_{21}^{(b)} x \mathcal{D}_{8+b} \otimes \mathcal{D}_{10-b} \otimes \mathcal{D}_{0}$; with $4 \leq b \leq 10, b \in \mathbb{Z}^{+}$

- $Z_{32} y Z_{31} z Z_{21}^{\left(b_{1}\right)} x Z_{21}^{\left(b_{2}\right)} x \mathcal{D}_{9+|b|} \otimes \mathcal{D}_{8-|b|} \otimes \mathcal{D}_{1}$; with $2 \leq|b|=b_{1}+b_{2} \leq 8$ and $b_{1} \geq 1$; $b_{1}, b_{2} \in \mathbb{Z}^{+}$

- $Z_{32}^{(2)} y z_{31} z Z_{21}^{\left(b_{1}\right)} x z_{21}^{\left(b_{2}\right)} x \mathcal{D}_{9+|b|} \otimes \mathcal{D}_{9-|b|} \otimes \mathcal{D}_{0}$; with $3 \leq|b|=b_{1}+b_{2} \leq 9$ and $b_{1} \geq 2$; $b_{1}, b_{2} \in \mathbb{Z}^{+}$

- $Z_{32} y Z_{32} y Z_{31} z Z_{21}^{(b)} x \mathcal{D}_{9+b} \otimes \mathcal{D}_{9-b} \otimes \mathcal{D}_{0}$; with $2 \leq b \leq 9, b \in \mathbb{Z}^{+}$ 
- In dimension five $\left(x_{5}\right)$ we have the sum of the following terms:

- $Z_{21}^{\left(b_{1}\right)} x Z_{21}^{\left(b_{2}\right)} x Z_{21}^{\left(b_{3}\right)} x Z_{21}^{\left(b_{4}\right)} x Z_{21}^{\left(b_{5}\right)} x \mathcal{D}_{8+|b|} \otimes \mathcal{D}_{7-|b|}$ $\otimes \mathcal{D}_{3} ;$ with $5 \leq|b|=\sum_{i=1}^{5} b_{i} \leq 7$ and $b_{1} \geq 1$; $b_{1}, b_{2}, b_{3}, b_{4}, b_{5} \in \mathbb{Z}^{+}$

- $z_{32} y Z_{21}^{\left(b_{1}\right)} x z_{21}^{\left(b_{2}\right)} x z_{21}^{\left(b_{3}\right)} x Z_{21}^{\left(b_{4}\right)} x \mathcal{D}_{8+|b|} \otimes \mathcal{D}_{8-|b|}$ $\otimes \mathcal{D}_{2} ;$ with $5 \leq|b|=\sum_{i=1}^{4} b_{i} \leq 8$ and $b_{1} \geq 2$; $b_{1}, b_{2}, b_{3}, b_{4} \in \mathbb{Z}^{+}$

- $Z_{32}^{(2)} y Z_{21}^{\left(b_{1}\right)} x Z_{21}^{\left(b_{2}\right)} x Z_{21}^{\left(b_{3}\right)} x Z_{21}^{\left(b_{4}\right)} x \mathcal{D}_{8+|b|} \otimes \mathcal{D}_{9-|b|}$ $\otimes \mathcal{D}_{1} ;$ with $6 \leq|b|=\sum_{i=1}^{4} b_{i} \leq 9$ and $b_{1} \geq 3$; $b_{1}, b_{2}, b_{3}, b_{4} \in \mathbb{Z}^{+}$

- $Z_{32} y Z_{32} y z_{21}^{\left(b_{1}\right)} x z_{21}^{\left(b_{2}\right)} x z_{21}^{\left(b_{3}\right)} x \mathcal{D}_{8+|b|} \otimes \mathcal{D}_{9-|b|} \otimes$ $\mathcal{D}_{1}$; with $5 \leq|b|=b_{1}+b_{2}+b_{3} \leq 9$ and $b_{1} \geq 3$; $b_{1}, b_{2}, b_{3} \in \mathbb{Z}^{+}$

- $Z_{32}^{(3)} y Z_{21}^{\left(b_{1}\right)} x Z_{21}^{\left(b_{2}\right)} x Z_{21}^{\left(b_{3}\right)} x Z_{21}^{\left(b_{4}\right)} x \mathcal{D}_{8+|b|} \otimes \mathcal{D}_{10-|b|}$ $\otimes \mathcal{D}_{0} ;$ with $7 \leq|b|=\sum_{i=1}^{4} b_{i} \leq 10$ and $b_{1} \geq 4$; $b_{1}, b_{2}, b_{3}, b_{4} \in \mathbb{Z}^{+}$

- $Z_{32}^{\left(c_{1}\right)} y Z_{32}^{\left(c_{2}\right)} y Z_{21}^{\left(b_{1}\right)} x Z_{21}^{\left(b_{2}\right)} x Z_{21}^{\left(b_{3}\right)} x \mathcal{D}_{8+|b|} \otimes$

$\mathcal{D}_{10-|b|} \otimes \mathcal{D}_{0} ;$ with $c_{1}+c_{2}=3$ and

$6 \leq|b|=b_{1}+b_{2}+b_{3} \leq 10$ and $b_{1} \geq 4$; $b_{1}, b_{2}, b_{3} \in \mathbb{Z}^{+}$

- $z_{32} y z_{32} y z_{32} y z_{21}^{\left(b_{1}\right)} x z_{21}^{\left(b_{2}\right)} x \mathcal{D}_{8+|b|} \otimes \mathcal{D}_{10-|b|}$ $\otimes \mathcal{D}_{0} ;$ with $5 \leq|b|=b_{1}+b_{2} \leq 10$ and $b_{1} \geq 4$; $b_{1}, b_{2} \in \mathbb{Z}^{+}$

- $Z_{32} y Z_{31} z Z_{21}^{\left(b_{1}\right)} x Z_{21}^{\left(b_{2}\right)} x Z_{21}^{\left(b_{3}\right)} x \mathcal{D}_{9+|b|} \otimes \mathcal{D}_{8-|b|} \otimes$ $\mathcal{D}_{1} ;$ with $3 \leq|b|=b_{1}+b_{2}+b_{3} \leq 8$ and $b_{1} \geq 1 ; b_{1}, b_{2}, b_{3} \in \mathbb{Z}^{+}$

- $Z_{32}^{(2)} y Z_{31} z Z_{21}^{\left(b_{1}\right)} x z_{21}^{\left(b_{2}\right)} x z_{21}^{\left(b_{3}\right)} x \mathcal{D}_{9+|b|} \otimes \mathcal{D}_{9-|b|} \otimes$ $\mathcal{D}_{0} ;$ with $4 \leq|b|=b_{1}+b_{2}+b_{3} \leq 9$ and $b_{1} \geq 2 ; b_{1}, b_{2}, b_{3} \in \mathbb{Z}^{+}$

- $Z_{32} y Z_{32} y Z_{31} z Z_{21}^{\left(b_{1}\right)} x Z_{21}^{\left(b_{2}\right)} x \mathcal{D}_{9+|b|} \otimes \mathcal{D}_{9-|b|} \otimes \mathcal{D}_{0}$; with $3 \leq|b|=b_{1}+b_{2} \leq 9$ and $b_{1} \geq 2$; $b_{1}, b_{2} \in \mathbb{Z}^{+}$

- In dimension six $\left(x_{6}\right)$ we have the sum of the following terms:

- $z_{21}^{\left(b_{1}\right)} x z_{21}^{\left(b_{2}\right)} x z_{21}^{\left(b_{3}\right)} x z_{21}^{\left(b_{4}\right)} x z_{21}^{\left(b_{5}\right)} x z_{21}^{\left(b_{6}\right)} x \mathcal{D}_{8+|b|}$ $\otimes \mathcal{D}_{7-|b|} \otimes \mathcal{D}_{3} ;$ with $6 \leq|b|=\sum_{i=1}^{6} b_{i} \leq 7$ and $b_{1} \geq 1 ; b_{1}, b_{2}, b_{3}, b_{4}, b_{5}, b_{6} \in \mathbb{Z}^{+}$

- $Z_{32} y Z_{21}^{\left(b_{1}\right)} x Z_{21}^{\left(b_{2}\right)} x Z_{21}^{\left(b_{3}\right)} x Z_{21}^{\left(b_{4}\right)} x z_{21}^{\left(b_{5}\right)} x \mathcal{D}_{8+|b|} \otimes$ $\mathcal{D}_{8-|b|} \otimes \mathcal{D}_{2} ;$ with $6 \leq|b|=\sum_{i=1}^{5} b_{i} \leq 8$ and $b_{1} \geq 2 ; b_{1}, b_{2}, b_{3}, b_{4}, b_{5} \in \mathbb{Z}^{+}$

- $z_{32}^{(2)}$ y $z_{21}^{\left(b_{1}\right)} x z_{21}^{\left(b_{2}\right)} x z_{21}^{\left(b_{3}\right)} x z_{21}^{\left(b_{4}\right)} x z_{21}^{\left(b_{5}\right)} x \mathcal{D}_{8+|b|}$ $\otimes \mathcal{D}_{9-|b|} \otimes \mathcal{D}_{1} ;$ with $7 \leq|b|=\sum_{i=1}^{5} b_{i} \leq 9$ and $b_{1} \geq 3 ; b_{1}, b_{2}, b_{3}, b_{4}, b_{5} \in \mathbb{Z}^{+}$

- $z_{32} y Z_{32} y z_{21}^{\left(b_{1}\right)} x z_{21}^{\left(b_{2}\right)} x z_{21}^{\left(b_{3}\right)} x z_{21}^{\left(b_{4}\right)} x \mathcal{D}_{8+|b|} \otimes$ $\mathcal{D}_{9-|b|} \otimes \mathcal{D}_{1} ;$ with $6 \leq|b|=\sum_{i=1}^{4} b_{i} \leq 9$ and $b_{1} \geq 3 ; b_{1}, b_{2}, b_{3}, b_{4} \in \mathbb{Z}^{+}$

- $z_{32}^{(3)} y Z_{21}^{\left(b_{1}\right)} x z_{21}^{\left(b_{2}\right)} x z_{21}^{\left(b_{3}\right)} x z_{21}^{\left(b_{4}\right)} x z_{21}^{\left(b_{5}\right)} x \mathcal{D}_{8+|b|} \otimes$ $\mathcal{D}_{10-|b|} \otimes \mathcal{D}_{0} ;$ with $8 \leq|b|=\sum_{i=1}^{5} b_{i} \leq 10$ and $b_{1} \geq 4 ; b_{1}, b_{2}, b_{3}, b_{4}, b_{5} \in \mathbb{Z}^{+}$
- $Z_{32}^{\left(c_{1}\right)} y Z_{32}^{\left(c_{2}\right)} y Z_{21}^{\left(b_{1}\right)} x Z_{21}^{\left(b_{2}\right)} x Z_{21}^{\left(b_{3}\right)} x Z_{21}^{\left(b_{4}\right)} x \mathcal{D}_{8+|b|}$ $\otimes \mathcal{D}_{10-|b|} \otimes \mathcal{D}_{0} ;$ with $c_{1}+c_{2}=3$ and $7 \leq|b|=\sum_{i=1}^{4} b_{i} \leq 10$ and $b_{1} \geq 4$; $b_{1}, b_{2}, b_{3}, b_{4}, c_{1}, c_{2} \in \mathbb{Z}^{+}$

- $z_{32} y z_{32} y z_{32} y z_{21}^{\left(b_{1}\right)} x z_{21}^{\left(b_{2}\right)} x z_{21}^{\left(b_{3}\right)} x \mathcal{D}_{8+|b|} \otimes$ $\mathcal{D}_{10-|b|} \otimes \mathcal{D}_{0} ;$ with $6 \leq|b|=b_{1}+b_{2}+b_{3} \leq 10$ and $b_{1} \geq 4 ; b_{1}, b_{2}, b_{3} \in \mathbb{Z}^{+}$

- $Z_{32} y Z_{31} z Z_{21}^{\left(b_{1}\right)} x Z_{21}^{\left(b_{2}\right)} x Z_{21}^{\left(b_{3}\right)} x Z_{21}^{\left(b_{4}\right)} x \mathcal{D}_{9+|b|} \otimes$ $\mathcal{D}_{8-|b|} \otimes \mathcal{D}_{1} ;$ with $4 \leq|b|=\sum_{i=1}^{4} b_{i} \leq 8$ and $b_{1} \geq 1 ; b_{1}, b_{2}, b_{3}, b_{4} \in \mathbb{Z}^{+}$

- $z_{32}^{(2)} y z_{31} z Z_{21}^{\left(b_{1}\right)} x z_{21}^{\left(b_{2}\right)} x z_{21}^{\left(b_{3}\right)} x z_{21}^{\left(b_{4}\right)} x \mathcal{D}_{9+|b|} \otimes$ $\mathcal{D}_{9-|b|} \otimes \mathcal{D}_{0} ;$ with $5 \leq|b|=\sum_{i=1}^{4} b_{i} \leq 9$ and $b_{1} \geq 2 ; b_{1}, b_{2}, b_{3}, b_{4} \in \mathbb{Z}^{+}$

- $z_{32} y z_{32} y z_{31} z z_{21}^{\left(b_{1}\right)} x z_{21}^{\left(b_{2}\right)} x z_{21}^{\left(b_{3}\right)} x \mathcal{D}_{9+|b|} \otimes$ $\mathcal{D}_{9-|b|} \otimes \mathcal{D}_{0} ;$ with $4 \leq|b|=b_{1}+b_{2}+b_{3} \leq 9$ and $b_{1} \geq 2 ; b_{1}, b_{2}, b_{3} \in \mathbb{Z}^{+}$

- In dimension seven $\left(x_{7}\right)$ we have the sum of the following terms:

- $Z_{21} x Z_{21} x Z_{21} x Z_{21} x Z_{21} x Z_{21} x Z_{21} x \mathcal{D}_{15} \otimes \mathcal{D}_{0} \otimes \mathcal{D}_{3}$

- $z_{32} y z_{21}^{\left(b_{1}\right)} x Z_{21}^{\left(b_{2}\right)} x z_{21}^{\left(b_{3}\right)} x z_{21}^{\left(b_{4}\right)} x z_{21}^{\left(b_{5}\right)} x z_{21}^{\left(b_{6}\right)} x$ $\mathcal{D}_{8+|b|} \otimes \mathcal{D}_{8-|b|} \otimes \mathcal{D}_{2} ;$ with $7 \leq|b|=\sum_{i=1}^{6} b_{i} \leq 8$ and $b_{1} \geq 2 ; b_{1}, b_{2}, b_{3}, b_{4}, b_{5}, b_{6} \in \mathbb{Z}^{+}$

- $z_{32} y z_{32} y z_{21}^{\left(b_{1}\right)} x z_{21}^{\left(b_{2}\right)} x z_{21}^{\left(b_{3}\right)} x z_{21}^{\left(b_{4}\right)} x z_{21}^{\left(b_{5}\right)} x$ $\mathcal{D}_{8+|b|} \otimes \mathcal{D}_{9-|b|} \otimes \mathcal{D}_{1} ;$ with $7 \leq|b|=\sum_{i=1}^{5} b_{i} \leq 9$ and $b_{1} \geq 3 ; b_{1}, b_{2}, b_{3}, b_{4}, b_{5} \in \mathbb{Z}^{+}$

- $z_{32}^{(2)} y Z_{21}^{\left(b_{1}\right)} x z_{21}^{\left(b_{2}\right)} x z_{21}^{\left(b_{3}\right)} x z_{21}^{\left(b_{4}\right)} x z_{21}^{\left(b_{5}\right)} x z_{21}^{\left(b_{6}\right)} x$

$\mathcal{D}_{8+|b|} \otimes \mathcal{D}_{9-|b|} \otimes \mathcal{D}_{1} ;$ with $8 \leq|b|=\sum_{i=1}^{6} b_{i} \leq 9$ and $b_{1} \geq 3 ; b_{1}, b_{2}, b_{3}, b_{4}, b_{5}, b_{6} \in \mathbb{Z}^{+}$

- $z_{32} y z_{32} y z_{32} y z_{21}^{\left(b_{1}\right)} x z_{21}^{\left(b_{2}\right)} x z_{21}^{\left(b_{3}\right)} x z_{21}^{\left(b_{4}\right)} x \mathcal{D}_{8+|b|}$ $\otimes \mathcal{D}_{10-|b|} \otimes \mathcal{D}_{1} ;$ with $7 \leq|b|=\sum_{i=1}^{4} b_{i} \leq 10$ and $b_{1} \geq 4 ; b_{1}, b_{2}, b_{3}, b_{4} \in \mathbb{Z}^{+}$

- $z_{32}^{\left(c_{1}\right)} y z_{32}^{\left(c_{2}\right)} y z_{21}^{\left(b_{1}\right)} x z_{21}^{\left(b_{2}\right)} x z_{21}^{\left(b_{3}\right)} x z_{21}^{\left(b_{4}\right)} x z_{21}^{\left(b_{5}\right)} x$ $\mathcal{D}_{8+|b|} \otimes \mathcal{D}_{10-|b|} \otimes \mathcal{D}_{0} ;$ with $c_{1}+c_{2}=3$ and $8 \leq|b|=\sum_{i=1}^{5} b_{i} \leq 10$ and $b_{1} \geq 4$;

$b_{1}, b_{2}, b_{3}, b_{4}, b_{5}, c_{1}, c_{2} \in \mathbb{Z}^{+}$

- $z_{32}^{(3)} y z_{21}^{\left(b_{1}\right)} x z_{21}^{\left(b_{2}\right)} x z_{21}^{\left(b_{3}\right)} x z_{21}^{\left(b_{4}\right)} x z_{21}^{\left(b_{5}\right)} x z_{21}^{\left(b_{6}\right)} x$ $\mathcal{D}_{8+|b|} \otimes \mathcal{D}_{10-|b|} \otimes \mathcal{D}_{0} ;$ with $9 \leq|b|=\sum_{i=1}^{6} b_{i} \leq$ 10 and $b_{1} \geq 4 ; b_{1}, b_{2}, b_{3}, b_{4}, b_{5}, b_{6} \in \mathbb{Z}^{+}$

- $z_{32} y z_{31} z z_{21}^{\left(b_{1}\right)} x z_{21}^{\left(b_{2}\right)} x z_{21}^{\left(b_{3}\right)} x z_{21}^{\left(b_{4}\right)} x z_{21}^{\left(b_{5}\right)} x \mathcal{D}_{9+|b|}$ $\otimes \mathcal{D}_{8-|b|} \otimes \mathcal{D}_{1} ;$ with $5 \leq|b|=\sum_{i=1}^{5} b_{i} \leq 8$ and $b_{1} \geq 1 ; b_{1}, b_{2}, b_{3}, b_{4}, b_{5} \in \mathbb{Z}^{+}$

- $z_{32} y Z_{32} y Z_{31} z Z_{21}^{\left(b_{1}\right)} x z_{21}^{\left(b_{2}\right)} x z_{21}^{\left(b_{3}\right)} x z_{21}^{\left(b_{4}\right)} x \mathcal{D}_{9+|b|}$ $\otimes \mathcal{D}_{9-|b|} \otimes \mathcal{D}_{0} ;$ with $5 \leq|b|=\sum_{i=1}^{4} b_{i} \leq 9$ and $b_{1} \geq 2 ; b_{1}, b_{2}, b_{3}, b_{4} \in \mathbb{Z}^{+}$

- $z_{32}^{(2)} y z_{31} z z_{21}^{\left(b_{1}\right)} x z_{21}^{\left(b_{2}\right)} x z_{21}^{\left(b_{3}\right)} x z_{21}^{\left(b_{4}\right)} x z_{21}^{\left(b_{5}\right)} x$

$\mathcal{D}_{9+|b|} \otimes \mathcal{D}_{9-|b|} \otimes \mathcal{D}_{0} ;$ with

$6 \leq|b|=\sum_{i=1}^{5} b_{i} \leq 9$ and $b_{1} \geq 2$;

$b_{1}, b_{2}, b_{3}, b_{4}, b_{5} \in \mathbb{Z}^{+}$ 
- In dimension eight $\left(X_{8}\right)$ we have the sum of the following terms:

- $Z_{32} y Z_{21} x Z_{21} x Z_{21} x Z_{21} x Z_{21} x Z_{21} x Z_{21} x \mathcal{D}_{16}$ $\otimes \mathcal{D}_{0} \otimes \mathcal{D}_{2}$

- $Z_{32} y Z_{32} y Z_{21}^{\left(b_{1}\right)} x Z_{21}^{\left(b_{2}\right)} x Z_{21}^{\left(b_{3}\right)} x Z_{21}^{\left(b_{4}\right)} x Z_{21}^{\left(b_{5}\right)} x$ $z_{21}^{\left(b_{6}\right)} x \mathcal{D}_{8+|b|} \otimes \mathcal{D}_{9-|b|} \otimes \mathcal{D}_{2} ;$ with

$8 \leq|b|=\sum_{i=1}^{6} b_{i} \leq 9$ and $b_{1} \geq 3$;

$b_{1}, b_{2}, b_{3}, b_{4}, b_{5}, b_{6} \in \mathbb{Z}^{+}$

- $Z_{32}^{(2)} y Z_{21}^{(3)} x z_{21} x z_{21} x z_{21} x z_{21} x z_{21} x z_{21} x D_{17}$ $\otimes \mathcal{D}_{0} \otimes \mathcal{D}_{1}$

- $z_{32} y z_{32} y z_{32} y z_{21}^{\left(b_{1}\right)} x z_{21}^{\left(b_{2}\right)} x z_{21}^{\left(b_{3}\right)} x z_{21}^{\left(b_{4}\right)} x z_{21}^{\left(b_{5}\right)} x$

$\mathcal{D}_{8+|b|} \otimes \mathcal{D}_{10-|b|} \otimes \mathcal{D}_{0} ;$ with $8 \leq|b|=\sum_{i=1}^{5} b_{i} \leq 10$ and $b_{1} \geq 4 ; b_{1}, b_{2}, b_{3}, b_{4}, b_{5} \in \mathbb{Z}^{+}$

- $z_{32}^{\left(c_{1}\right)} y z_{32}^{\left(c_{2}\right)} y z_{21}^{\left(b_{1}\right)} x z_{21}^{\left(b_{2}\right)} x z_{21}^{\left(b_{3}\right)} x z_{21}^{\left(b_{4}\right)} x z_{21}^{\left(b_{5}\right)} x$ $z_{21}^{\left(b_{6}\right)} x \mathcal{D}_{8+|b|} \otimes \mathcal{D}_{10-|b|} \otimes \mathcal{D}_{0} ;$ with $c_{1}+c_{2}=3$ and $9 \leq|b|=\sum_{i=1}^{6} b_{i} \leq 10$ and $b_{1} \geq 4$; $b_{1}, b_{2}, b_{3}, b_{4}, b_{5}, b_{6}, c_{1}, c_{2} \in \mathbb{Z}^{+}$

- $Z_{32}^{(3)} y Z_{21} x Z_{21} x Z_{21} x Z_{21} x Z_{21} x Z_{21} x Z_{21} x \mathcal{D}_{15} \otimes$ $\mathcal{D}_{2} \otimes \mathcal{D}_{1}$

- $Z_{32} y Z_{31} z Z_{21}^{\left(b_{1}\right)} x z_{21}^{\left(b_{2}\right)} x Z_{21}^{\left(b_{3}\right)} x Z_{21}^{\left(b_{4}\right)} x z_{21}^{\left(b_{5}\right)} x$ $Z_{21}^{\left(b_{6}\right)} x \mathcal{D}_{9+|b|} \otimes \mathcal{D}_{8-|b|} \otimes \mathcal{D}_{1} ;$ with $7 \leq|b|=\sum_{i=1}^{6} b_{i} \leq 8$ and $b_{1} \geq 2$; $b_{1}, b_{2}, b_{3}, b_{4}, b_{5}, b_{6} \in \mathbb{Z}^{+}$

- $z_{32} y Z_{32} y Z_{31} z z_{21}^{\left(b_{1}\right)} x z_{21}^{\left(b_{2}\right)} x z_{21}^{\left(b_{3}\right)} x z_{21}^{\left(b_{4}\right)} x$ $z_{21}^{\left(b_{5}\right)} x \mathcal{D}_{9+|b|} \otimes \mathcal{D}_{9-|b|} \otimes \mathcal{D}_{0} ;$ with $6 \leq|b|=\sum_{i=1}^{5} b_{i} \leq 9$ and $b_{1} \geq 2$; $b_{1}, b_{2}, b_{3}, b_{4}, b_{5} \in \mathbb{Z}^{+}$

- $z_{32}^{(2)} y z_{31} z z_{21}^{\left(b_{1}\right)} x z_{21}^{\left(b_{2}\right)} x z_{21}^{\left(b_{3}\right)} x z_{21}^{\left(b_{4}\right)} x z_{21}^{\left(b_{5}\right)} x$ $z_{21}^{\left(b_{6}\right)} x \mathcal{D}_{9+|b|} \otimes \mathcal{D}_{9-|b|} \otimes \mathcal{D}_{0} ;$ with

$7 \leq|b|=\sum_{i=1}^{6} b_{i} \leq 9$ and $b_{1} \geq 2$;

$b_{1}, b_{2}, b_{3}, b_{4}, b_{5}, b_{6} \in \mathbb{Z}^{+}$

- In dimension eight $\left(X_{9}\right)$ we have the sum of the following terms:

- $z_{32} y z_{32} y z_{21}^{(3)} x z_{21} x z_{21} x z_{21} x z_{21} x z_{21} x$ $z_{21} x \mathcal{D}_{17} \otimes \mathcal{D}_{0} \otimes \mathcal{D}_{1}$

- $z_{32} y z_{32} y z_{32} y z_{21}^{\left(b_{1}\right)} x z_{21}^{\left(b_{2}\right)} x z_{21}^{\left(b_{3}\right)} x z_{21}^{\left(b_{4}\right)} x$ $Z_{21}^{\left(b_{5}\right)} x Z_{21}^{\left(b_{6}\right)} x \mathcal{D}_{8+|b|} \otimes \mathcal{D}_{10-|b|} \otimes \mathcal{D}_{0}$; with $9 \leq|b|=\sum_{i=1}^{6} b_{i} \leq 10$ and $b_{1} \geq 4$; $b_{1}, b_{2}, b_{3}, b_{4}, b_{5}, b_{6} \in \mathbb{Z}^{+}$

- $z_{32}^{\left(c_{1}\right)} y z_{32}^{\left(c_{2}\right)} y z_{21}^{(4)} x z_{21} x z_{21} x z_{21} x z_{21} x z_{21} x$ $z_{21} x \mathcal{D}_{18} \otimes \mathcal{D}_{0} \otimes \mathcal{D}_{0} ;$ with $c_{1}+c_{2}=3$

- $z_{32} y z_{31} z z_{21}^{\left(b_{1}\right)} x z_{21}^{\left(b_{2}\right)} x z_{21}^{\left(b_{3}\right)} x z_{21}^{\left(b_{4}\right)} x z_{21}^{\left(b_{5}\right)} x$ $z_{21}^{\left(b_{6}\right)} x Z_{21}^{\left(b_{7}\right)} x \mathcal{D}_{9+|b|} \otimes \mathcal{D}_{8-|b|} \otimes \mathcal{D}_{1}$; with $7 \leq|b|=\sum_{i=1}^{6} b_{i} \leq 8$ and $b_{1} \geq 1$; $b_{1}, b_{2}, b_{3}, b_{4}, b_{5}, b_{6}, b_{7} \in \mathbb{Z}^{+}$

- $z_{32} y z_{32} y z_{31} z z_{21}^{\left(b_{1}\right)} x z_{21}^{\left(b_{2}\right)} x z_{21}^{\left(b_{3}\right)} x z_{21}^{\left(b_{4}\right)} x$ $z_{21}^{\left(b_{5}\right)} x z_{21}^{\left(b_{6}\right)} x \mathcal{D}_{9+|b|} \otimes \mathcal{D}_{9-|b|} \otimes \mathcal{D}_{0}$; with $7 \leq|b|=\sum_{i=1}^{5} b_{i} \leq 9$ and $b_{1} \geq 2$; $b_{1}, b_{2}, b_{3}, b_{4}, b_{5}, b_{6} \in \mathbb{Z}^{+}$
- $z_{32}^{(2)} y z_{31} z z_{21}^{\left(b_{1}\right)} x z_{21}^{\left(b_{2}\right)} x z_{21}^{\left(b_{3}\right)} x z_{21}^{\left(b_{4}\right)} x z_{21}^{\left(b_{5}\right)} x$ $z_{21}^{\left(b_{6}\right)} x z_{21}^{\left(b_{7}\right)} x \mathcal{D}_{9+|b|} \otimes \mathcal{D}_{9-|b|} \otimes \mathcal{D}_{0}$; with $8 \leq|b|=\sum_{i=1}^{7} b_{i} \leq 9$ and $b_{1} \geq 1$;

$b_{1}, b_{2}, b_{3}, b_{4}, b_{5}, b_{6}, b_{7} \in \mathbb{Z}^{+}$

- In dimension eight $\left(x_{10}\right)$ we have the sum of the following terms:

- $z_{32} y z_{32} y z_{32} y z_{21}^{(4)} x z_{21} x z_{21} x z_{21} x z_{21} x$ $z_{21} x Z_{21} x \mathcal{D}_{18} \otimes \mathcal{D}_{0} \otimes \mathcal{D}_{0}$

- $z_{32} y z_{31} z z_{21} x z_{21} x z_{21} x z_{21} x z_{21} x z_{21} x$ $z_{21} x Z_{21} x \mathcal{D}_{17} \otimes \mathcal{D}_{0} \otimes \mathcal{D}_{1}$

- $z_{32} y z_{32} y z_{31} z z_{21}^{\left(b_{1}\right)} x z_{21}^{\left(b_{2}\right)} x z_{21}^{\left(b_{3}\right)} x z_{21}^{\left(b_{4}\right)} x$ $z_{21}^{\left(b_{5}\right)} x Z_{21}^{\left(b_{6}\right)} x Z_{21}^{\left(b_{7}\right)} x \mathcal{D}_{9+|b|} \otimes \mathcal{D}_{9-|b|} \otimes \mathcal{D}_{0}$; with $8 \leq|b|=\sum_{i=1}^{5} b_{i} \leq 9$ and $b_{1} \geq 2$;

$b_{1}, b_{2}, b_{3}, b_{4}, b_{5}, b_{6}, b_{7} \in \mathbb{Z}^{+}$

- $z_{32}^{(2)} y z_{31} z z_{21}^{(2)} x z_{21} x z_{21} x z_{21} x z_{21} x z_{21} x$ $z_{21} x z_{21} x z_{21} x \mathcal{D}_{18} \otimes \mathcal{D}_{0} \otimes \mathcal{D}_{0}$

Finally In dimension nine $\left(\chi_{11}\right)$ we have

- $z_{32} y z_{32} y z_{31} z z_{21}^{(2)} x z_{21} x z_{21} x z_{21} x z_{21} x$ $z_{21} x Z_{21} x Z_{21} x \mathcal{D}_{18} \otimes \mathcal{D}_{0} \otimes \mathcal{D}_{0}$

\section{Lascoux Resolution of the Partition $(8,7,3)$}

The Lascoux resolution of the Weyl module associated to the partition $(8,7,3)$ is

$$
\mathcal{D}_{10} \mathcal{F} \otimes \mathcal{D}_{7} \mathcal{F} \otimes \mathcal{D}_{1} \mathcal{F} \longrightarrow \begin{gathered}
\mathcal{D}_{10} \mathcal{F} \otimes \mathcal{D}_{6} \mathcal{F} \otimes \mathcal{D}_{2} \mathcal{F} \\
\mathcal{D}_{9} \mathcal{F} \otimes \mathcal{D}_{8} \mathcal{F} \otimes \mathcal{D}_{1} \mathcal{F}
\end{gathered}
$$

$$
\longrightarrow \begin{aligned}
& \mathcal{D}_{9} \mathcal{F} \otimes \mathcal{D}_{6} \mathcal{F} \otimes \mathcal{D}_{3} \mathcal{F} \\
& \mathcal{D}_{8} \mathcal{F} \otimes \mathcal{D}_{8} \mathcal{F} \otimes \mathcal{D}_{2} \mathcal{F}
\end{aligned} \mathcal{D}_{8} \mathcal{F} \otimes \mathcal{D}_{7} \mathcal{F} \otimes \mathcal{D}_{3} \mathcal{F}
$$

Where the position of the terms of the complex determined by the length of the permutations to which they correspond.

Then the Lascaux complex has the correspondence between its terms as pursue:

$\mathcal{D}_{8} \mathcal{F} \otimes \mathcal{D}_{7} \mathcal{F} \otimes \mathcal{D}_{3} \mathcal{F} \leftrightarrow$ identity

$\mathcal{D}_{9} \mathcal{F} \otimes \mathcal{D}_{6} \mathcal{F} \otimes \mathcal{D}_{3} \mathcal{F} \leftrightarrow$ (12)

$\mathcal{D}_{8} \mathcal{F} \otimes \mathcal{D}_{8} \mathcal{F} \otimes \mathcal{D}_{2} \mathcal{F} \leftrightarrow(23)$

$\mathcal{D}_{10} \mathcal{F} \otimes \mathcal{D}_{6} \mathcal{F} \otimes \mathcal{D}_{2} \mathcal{F} \leftrightarrow(123)$

$\mathcal{D}_{9} \mathcal{F} \otimes \mathcal{D}_{8} \mathcal{F} \otimes \mathcal{D}_{1} \mathcal{F} \leftrightarrow(132)$

$\mathcal{D}_{10} \mathcal{F} \otimes \mathcal{D}_{7} \mathcal{F} \otimes \mathcal{D}_{1} \mathcal{F} \leftrightarrow(13)$

As in (8) the terms can be exhibit as pursue $x_{0}=\mathcal{L}_{0}=\mathcal{M}_{0}$

$x_{1}=\mathcal{L}_{1} \oplus \mathcal{M}_{1}$

$x_{2}=\mathcal{L}_{2} \oplus \mathcal{M}_{2}$

$x_{3}=\mathcal{L}_{3} \oplus \mathcal{M}_{3}$

$x_{j}=\mathcal{M}_{j} \quad$ for $j=4,5, \ldots, 11$

Where $\mathcal{L}_{e}$ are the sum of the Lascoux terms and $\mathcal{M}_{e}$ are the sum of the others. pursue

Now, we acquaint the map $\sigma_{1}: \mathcal{M}_{1} \longrightarrow \mathcal{L}_{1}$ as 
- $z_{21}^{(2)} x(v) \mapsto \frac{1}{2} z_{21} x \partial_{21}(v) ;$ where $v \in \mathcal{D}_{10} \otimes \mathcal{D}_{5} \otimes \mathcal{D}_{3}$

- $Z_{21}^{(3)} x(v) \mapsto \frac{1}{3} Z_{21} x \partial_{21}^{(2)}(v)$; where $v \in \mathcal{D}_{11} \otimes \mathcal{D}_{4} \otimes \mathcal{D}_{3}$

- $Z_{21}^{(4)} x(v) \mapsto \frac{1}{4} Z_{21} x \partial_{21}^{(3)}(v)$; where $v \in \mathcal{D}_{12} \otimes \mathcal{D}_{3} \otimes \mathcal{D}_{3}$ - $Z_{21}^{(5)} x(v) \mapsto \frac{1}{5} z_{21} x \partial_{21}^{(4)}(v)$; where $v \in \mathcal{D}_{13} \otimes \mathcal{D}_{2} \otimes \mathcal{D}_{3}$ - $Z_{21}^{(6)} x(v) \mapsto \frac{1}{6} z_{21} x \partial_{21}^{(5)}(v)$; where $v \in \mathcal{D}_{14} \otimes \mathcal{D}_{1} \otimes \mathcal{D}_{3}$ - $Z_{21}^{(7)} x(v) \mapsto \frac{1}{7} z_{21} x \partial_{21}^{(6)}(v)$; where $v \in \mathcal{D}_{15} \otimes \mathcal{D}_{0} \otimes \mathcal{D}_{3}$ - $Z_{32}^{(2)} y(v) \mapsto \frac{1}{2} Z_{32} y \partial_{32}(v)$; where $v \in \mathcal{D}_{8} \otimes \mathcal{D}_{9} \otimes \mathcal{D}_{1}$

- $z_{32}^{(3)} y(v) \mapsto \frac{1}{3} z_{32} x \partial_{32}^{(2)}(v)$; where $v \in \mathcal{D}_{8} \otimes \mathcal{D}_{10} \otimes \mathcal{D}_{0}$

We ought to indicate that the map $\sigma_{1}$ implement the identity

$$
\delta_{\mathcal{L}_{1} \mathcal{L}_{0}} \sigma_{1}=\delta_{\mathcal{M}_{1} \mathcal{M}_{0}}
$$

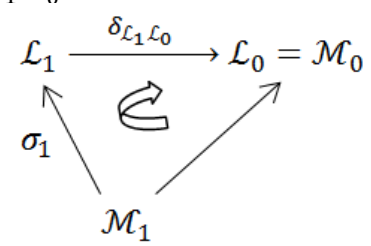

Where $\delta_{\mathcal{L}_{1} \mathcal{L}_{0}}$ the component of the boundary of the fat complex which conveys $\mathcal{L}_{1}$ to $\mathcal{L}_{0}$.

We employ the registration $\delta_{\mathcal{L}_{t+1} \mathcal{L}_{t}}, \delta_{\mathcal{L}_{t+1} \mathcal{M}_{1}}$ etc.

Thus we can acquaint $\partial_{1}: \mathcal{L}_{1} \stackrel{\mathcal{L}^{2}+1}{\longrightarrow} \mathcal{L}_{0}$ as

$$
\partial_{1}=\delta_{\mathcal{L}_{1} \mathcal{L}_{0}}
$$

It is plainsman to exhibit that $\partial_{1}$ implement 2 , for example we adopt one of them:

$$
\begin{aligned}
& \left(\delta_{\mathcal{L}_{1} \mathcal{L}_{0}} \circ \sigma_{1}\right)\left(z_{21}^{(5)} x(v)\right)= \\
& \delta_{\mathcal{L}_{1} \mathcal{L}_{0}} \circ \sigma_{1}\left(\frac{1}{5} z_{21} x \partial_{21}^{(4)}(v)\right) \\
& =\frac{1}{5}\left(\partial_{21} \partial_{21}^{(4)}(v)\right)=\partial_{21}^{(5)}(v)=\delta_{\mathcal{M}_{1} \mathcal{M}_{0}}\left(z_{21}^{(5)} x(v)\right)
\end{aligned}
$$

As long as we can acquaint $\partial_{2}: \mathcal{L}_{2} \longrightarrow \mathcal{L}_{1}$ by $\partial_{2}=\delta_{\mathcal{L}_{2} \mathcal{L}_{1}}+\sigma_{1} \circ \delta_{\mathcal{L}_{2} \mathcal{M}_{1}}$

Proposition 3.1: (4), (5) and (8)

Proof:

The composition $\partial_{1} \circ \partial_{2}$ equal to zero.

$$
\begin{aligned}
\partial_{1} \circ \partial_{2}(g)= & \delta_{\mathcal{L}_{1} \mathcal{L}_{0}} \circ\left(\delta_{\mathcal{L}_{2} \mathcal{L}_{1}}(g)+\sigma_{1} \delta_{\mathcal{L}_{2} \mathcal{M}_{1}}(g)\right) \\
= & \delta_{\mathcal{L}_{1} \mathcal{L}_{0}} \circ \delta_{\mathcal{L}_{2} \mathcal{L}_{1}}(g)+ \\
& \delta_{\mathcal{L}_{1} \mathcal{L}_{0}} \circ \sigma_{1} \delta_{\mathcal{L}_{2} \mathcal{M}_{1}}(g)
\end{aligned}
$$

But $\delta_{\mathcal{L}_{1} \mathcal{L}_{0}} \sigma_{1}=\delta_{\mathcal{M}_{1} \mathcal{M}_{0}}$. Then we attain

$\partial_{1} \circ \partial_{2}(g)=\delta_{\mathcal{L}_{1} \mathcal{L}_{0}} \circ \delta_{\mathcal{L}_{2} \mathcal{L}_{1}}(g)+\delta_{\mathcal{M}_{1} \mathcal{M}_{0}} \delta_{\mathcal{L}_{2} \mathcal{M}_{1}}(g)$

Which equal to zero, because of the properties of the boundary map $\delta$, so we attain $\partial_{1} \circ \partial_{2}=0$.
Now, we have to acquaint $\sigma_{2}: \mathcal{M}_{2} \longrightarrow \mathcal{L}_{2}$ such that

$$
\delta_{\mathcal{M}_{2} \mathcal{L}_{1}}+\sigma_{1} \circ \delta_{\mathcal{M}_{2} \mathcal{M}_{1}}=\left(\delta_{\mathcal{L}_{2} \mathcal{L}_{1}}+\sigma_{1} \circ \delta_{\mathcal{L}_{2} \mathcal{M}_{1}}\right) \circ \sigma_{2}
$$

We acquaint this map as pursue:

- $Z_{21} x Z_{21} x(v) \mapsto 0$; where $v \in \mathcal{D}_{10} \otimes \mathcal{D}_{5} \otimes \mathcal{D}_{3}$ - $Z_{21}^{(2)} x Z_{21} x(v) \mapsto 0 ;$ where $v \in \mathcal{D}_{11} \otimes \mathcal{D}_{4} \otimes \mathcal{D}_{3}$ - $Z_{21} x Z_{21}^{(2)} x(v) \mapsto 0$; where $v \in \mathcal{D}_{11} \otimes \mathcal{D}_{4} \otimes \mathcal{D}_{3}$ - $Z_{21}^{(3)} x Z_{21} x(v) \mapsto 0$; where $v \in \mathcal{D}_{12} \otimes \mathcal{D}_{3} \otimes \mathcal{D}_{3}$ - $Z_{21} x Z_{21}^{(3)} x(v) \mapsto 0$; where $v \in \mathcal{D}_{12} \otimes \mathcal{D}_{3} \otimes \mathcal{D}_{3}$ - $Z_{21}^{(2)} x Z_{21}^{(2)} x(v) \mapsto 0$; where $v \in \mathcal{D}_{12} \otimes \mathcal{D}_{3} \otimes \mathcal{D}_{3}$ - $Z_{21}^{(4)} x Z_{21} x(v) \mapsto 0$; where $v \in \mathcal{D}_{13} \otimes \mathcal{D}_{2} \otimes \mathcal{D}_{3}$ - $Z_{21} x Z_{21}^{(4)} x(v) \mapsto 0$; where $v \in \mathcal{D}_{13} \otimes \mathcal{D}_{2} \otimes \mathcal{D}_{3}$ - $Z_{21}^{(3)} x Z_{21}^{(2)} x(v) \mapsto 0$; where $v \in \mathcal{D}_{13} \otimes \mathcal{D}_{2} \otimes \mathcal{D}_{3}$ - $Z_{21}^{(2)} x Z_{21}^{(3)} x(v) \mapsto 0$; where $v \in \mathcal{D}_{13} \otimes \mathcal{D}_{2} \otimes \mathcal{D}_{3}$ - $Z_{21}^{(5)} x Z_{21} x(v) \mapsto 0$; where $v \in \mathcal{D}_{14} \otimes \mathcal{D}_{1} \otimes \mathcal{D}_{3}$ - $Z_{21} x Z_{21}^{(5)} x(v) \mapsto 0$; where $v \in \mathcal{D}_{14} \otimes \mathcal{D}_{1} \otimes \mathcal{D}_{3}$ - $Z_{21}^{(3)} x Z_{21}^{(3)} x(v) \mapsto 0$; where $v \in \mathcal{D}_{14} \otimes \mathcal{D}_{1} \otimes \mathcal{D}_{3}$ - $Z_{21}^{(4)} x Z_{21}^{(2)} x(v) \mapsto 0$; where $v \in \mathcal{D}_{14} \otimes \mathcal{D}_{1} \otimes \mathcal{D}_{3}$ - $Z_{21}^{(2)} x Z_{21}^{(4)} x(v) \mapsto 0$; where $v \in \mathcal{D}_{14} \otimes \mathcal{D}_{1} \otimes \mathcal{D}_{3}$ - $Z_{21}^{(6)} x Z_{21} x(v) \mapsto 0$; where $v \in \mathcal{D}_{15} \otimes \mathcal{D}_{0} \otimes \mathcal{D}_{3}$ - $Z_{21} x Z_{21}^{(6)} x(v) \mapsto 0$; where $v \in \mathcal{D}_{15} \otimes \mathcal{D}_{0} \otimes \mathcal{D}_{3}$ - $Z_{21}^{(5)} x Z_{21}^{(2)} x(v) \mapsto 0$; where $v \in \mathcal{D}_{15} \otimes \mathcal{D}_{0} \otimes \mathcal{D}_{3}$ - $Z_{21}^{(2)} x Z_{21}^{(5)} x(v) \mapsto 0$; where $v \in \mathcal{D}_{15} \otimes \mathcal{D}_{0} \otimes \mathcal{D}_{3}$ - $Z_{21}^{(4)} x Z_{21}^{(3)} x(v) \mapsto 0$; where $v \in \mathcal{D}_{15} \otimes \mathcal{D}_{0} \otimes \mathcal{D}_{3}$ - $Z_{21}^{(3)} x Z_{21}^{(4)} x(v) \mapsto 0$; where $v \in \mathcal{D}_{15} \otimes \mathcal{D}_{0} \otimes \mathcal{D}_{3}$ - $z_{32} y z_{21}^{(3)} x(v) \mapsto \frac{1}{3} z_{32} y z_{21}^{(2)} x \partial_{21}(v) ;$ where $v \in \mathcal{D}_{11} \otimes \mathcal{D}_{5} \otimes \mathcal{D}_{2}$

- $z_{32} y Z_{21}^{(4)} x(v) \mapsto \frac{1}{6} z_{32} y Z_{21}^{(2)} x \partial_{21}^{(2)}(v)$; where $v \in \mathcal{D}_{12} \otimes \mathcal{D}_{4} \otimes \mathcal{D}_{2}$

- $z_{32} y z_{21}^{(5)} x(v) \mapsto \frac{1}{10} z_{32} y z_{21}^{(2)} x \partial_{21}^{(3)}(v)$; where $v \in \mathcal{D}_{13} \otimes \mathcal{D}_{3} \otimes \mathcal{D}_{2}$

- $z_{32} y Z_{21}^{(6)} x(v) \mapsto \frac{1}{15} z_{32} y z_{21}^{(2)} x \partial_{21}^{(4)}(v)$; where $v \in \mathcal{D}_{14} \otimes \mathcal{D}_{2} \otimes \mathcal{D}_{2}$

- $z_{32} y z_{21}^{(7)} x(v) \mapsto \frac{1}{21} z_{32} y z_{21}^{(2)} x \partial_{21}^{(5)}(v)$; where $v \in \mathcal{D}_{15} \otimes \mathcal{D}_{1} \otimes \mathcal{D}_{2}$

- $z_{32} y z_{21}^{(8)} x(v) \mapsto \frac{1}{28} z_{32} y z_{21}^{(2)} x \partial_{21}^{(6)}(v)$; where $v \in \mathcal{D}_{16} \otimes \mathcal{D}_{0} \otimes \mathcal{D}_{2}$

- $Z_{32} y Z_{32} y(v) \mapsto 0$; where $v \in \mathcal{D}_{8} \otimes \mathcal{D}_{9} \otimes \mathcal{D}_{1}$ - $z_{32}^{(2)} y z_{21}^{(3)} x(v) \mapsto \frac{1}{3}\left(z_{32} y z_{21}^{(2)} x \partial_{31}(v)-\right.$ $\left.Z_{32} y Z_{31} z \partial_{21}^{(2)}(v)\right)$; where $v \in \mathcal{D}_{11} \otimes \mathcal{D}_{6} \otimes \mathcal{D}_{1}$ - $z_{32}^{(2)} y z_{21}^{(4)} x(v) \mapsto \frac{1}{12} z_{32} y z_{21}^{(2)} x \partial_{21} \partial_{31}(v)-$ $\frac{1}{4} Z_{32} y Z_{31} z \partial_{21}^{(3)}(v) ;$ where $v \in \mathcal{D}_{12} \otimes \mathcal{D}_{5} \otimes \mathcal{D}_{1}$ - $z_{32}^{(2)} y z_{21}^{(5)} x(v) \mapsto \frac{1}{30} z_{32} y z_{21}^{(2)} x \partial_{21}^{(2)} \partial_{31}(v)-$ $\frac{1}{5} Z_{32} y Z_{31} z \partial_{21}^{(4)}(v)$; where $v \in \mathcal{D}_{13} \otimes \mathcal{D}_{4} \otimes \mathcal{D}_{1}$ 
- $z_{32}^{(2)} y Z_{21}^{(6)} x(v) \mapsto \frac{1}{60} z_{32} y z_{21}^{(2)} x \partial_{21}^{(3)} \partial_{31}(v)-$ $\frac{1}{6} Z_{32} y Z_{31} z \partial_{21}^{(5)}(v)$; where $v \in \mathcal{D}_{14} \otimes \mathcal{D}_{3} \otimes \mathcal{D}_{1}$ - $Z_{32}^{(2)} y Z_{21}^{(7)} x(v) \mapsto \frac{1}{105} Z_{32} y Z_{21}^{(2)} x \partial_{21}^{(4)} \partial_{31}(v)-$ $\frac{1}{7} Z_{32} y Z_{31} z \partial_{21}^{(6)}(v) ;$ where $v \in \mathcal{D}_{15} \otimes \mathcal{D}_{2} \otimes \mathcal{D}_{1}$ - $Z_{32}^{(2)} y Z_{21}^{(8)} x(v) \mapsto \frac{1}{168} z_{32} y Z_{21}^{(2)} x \partial_{21}^{(5)} \partial_{31}(v)-$ $\frac{1}{8} Z_{32} y Z_{31} z \partial_{21}^{(7)}(v) ;$ where $v \in \mathcal{D}_{16} \otimes \mathcal{D}_{1} \otimes \mathcal{D}_{1}$ - $z_{32}^{(2)} y z_{21}^{(9)} x(v) \mapsto \frac{1}{252} z_{32} y z_{21}^{(2)} x \partial_{21}^{(6)} \partial_{31}(v)-$ $\frac{1}{9} Z_{32} y Z_{31} z \partial_{21}^{(7)}(v) ;$ where $v \in \mathcal{D}_{17} \otimes \mathcal{D}_{0} \otimes \mathcal{D}_{1}$ - $Z_{32}^{(2)} y Z_{32} y(v) \mapsto 0$; where $v \in \mathcal{D}_{8} \otimes \mathcal{D}_{10} \otimes \mathcal{D}_{0}$ - $Z_{32} y Z_{32}^{(2)} y(v) \mapsto 0$ where $v \in \mathcal{D}_{8} \otimes \mathcal{D}_{10} \otimes \mathcal{D}_{0}$ - $z_{32}^{(3)} y z_{21}^{(4)} x(v) \mapsto \frac{1}{3} z_{32} y z_{21}^{(2)} x \partial_{31}^{(2)}(v)-$ $\frac{1}{6} z_{32} y z_{21}^{(2)} x \partial_{21}^{(2)} \partial_{32}^{(2)}(v)-$ $\frac{1}{3} z_{32} y z_{31} z \partial_{21}^{(3)} \partial_{32}(v)$;

where $v \in \mathcal{D}_{12} \otimes \mathcal{D}_{6} \otimes \mathcal{D}_{0}$

- $z_{32}^{(3)} y z_{21}^{(5)} x(v) \mapsto \frac{1}{9} z_{32} y z_{21}^{(2)} x \partial_{21} \partial_{31}^{(2)}(v)-$ $\frac{7}{90} z_{32} y z_{21}^{(2)} x \partial_{21}^{(3)} \partial_{32}^{(2)}(v)-$ $\frac{2}{9} z_{32} y z_{31} z \partial_{21}^{(4)} \partial_{32}(v)$;

where $v \in \mathcal{D}_{13} \otimes \mathcal{D}_{5} \otimes \mathcal{D}_{0}$

- $z_{32}^{(3)} y z_{21}^{(6)} x(v) \mapsto \frac{1}{18} z_{32} y Z_{21}^{(2)} x \partial_{21}^{(2)} \partial_{31}^{(2)}(v)-$ $\frac{2}{45} z_{32} y z_{21}^{(2)} x \partial_{21}^{(4)} \partial_{32}^{(2)}(v)-$ $\frac{1}{6} z_{32} y z_{31} z \partial_{21}^{(5)} \partial_{32}(v)$;

where $v \in \mathcal{D}_{14} \otimes \mathcal{D}_{4} \otimes \mathcal{D}_{0}$

- $z_{32}^{(3)} y z_{21}^{(7)} x(v) \mapsto \frac{1}{30} z_{32} y z_{21}^{(2)} x \partial_{21}^{(3)} \partial_{31}^{(2)}(v)-$ $\frac{1}{35} z_{32} y z_{21}^{(2)} x \partial_{21}^{(5)} \partial_{32}^{(2)}(v)-$ $\frac{2}{15} z_{32} y z_{31} z \partial_{21}^{(6)} \partial_{32}(v)$;

where $v \in \mathcal{D}_{15} \otimes \mathcal{D}_{3} \otimes \mathcal{D}_{0}$

- $Z_{32}^{(3)} y z_{21}^{(8)} x(v) \mapsto \frac{1}{45} z_{32} y Z_{21}^{(2)} x \partial_{21}^{(4)} \partial_{31}^{(2)}(v)-$ $\frac{5}{252} z_{32} y z_{21}^{(2)} x \partial_{21}^{(6)} \partial_{32}^{(2)}(v)-$ $\frac{1}{9} z_{32} y z_{31} z \partial_{21}^{(7)} \partial_{32}(v)$;

where $v \in \mathcal{D}_{16} \otimes \mathcal{D}_{2} \otimes \mathcal{D}_{0}$

- $Z_{32}^{(3)} y Z_{21}^{(9)} x(v) \mapsto \frac{1}{63} z_{32} y z_{21}^{(2)} x \partial_{21}^{(5)} \partial_{31}^{(2)}(v)+$ $\frac{1}{84} z_{32} y z_{21}^{(2)} x \partial_{21}^{(6)} \partial_{32} \partial_{31}(v)$;

where $v \in \mathcal{D}_{17} \otimes \mathcal{D}_{1} \otimes \mathcal{D}_{0}$

- $z_{32}^{(3)} y z_{21}^{(10)} x(v) \mapsto \frac{1}{84} z_{32} y z_{21}^{(2)} x \partial_{21}^{(6)} \partial_{31}^{(2)}(v)$; where $v \in \mathcal{D}_{18} \otimes \mathcal{D}_{0} \otimes \mathcal{D}_{0}$

- $z_{32}^{(2)} y z_{31} z(v) \mapsto \frac{1}{3} z_{32} y z_{31} z \partial_{32}(v)$;

where $v \in \mathcal{D}_{9} \otimes \mathcal{D}_{9} \otimes \mathcal{D}_{0}$

It is plainsman to exhibit that $\sigma_{2}$ which is acquainting above implement 3 , for example we adopt one of them:

Where $v \in \mathcal{D}_{15} \otimes \mathcal{D}_{2} \otimes \mathcal{D}_{1}$

$\left(\delta_{\mathcal{M}_{2} \mathcal{L}_{1}}+\sigma_{1} \circ \delta_{\mathcal{M}_{2} \mathcal{M}_{1}}\right)\left(Z_{32}^{(2)} y Z_{21}^{(7)} x(v)\right)$

$$
\begin{aligned}
= & \sigma_{1}\left(Z_{21}^{(7)} x \partial_{32}^{(2)}(v)+z_{21}^{(6)} x \partial_{32} \partial_{31}(v)+\right. \\
& \left.z_{21}^{(5)} x \partial_{31}^{(2)}(v)-z_{32}^{(2)} y \partial_{21}^{(7)}(v)\right) \\
= & \frac{1}{7} z_{21} x \partial_{21}^{(6)} \partial_{32}^{(2)}(v)+\frac{1}{6} z_{21} x \partial_{21}^{(5)} \partial_{32} \partial_{31}(v)+ \\
& \frac{1}{5} z_{21} x \partial_{21}^{(4)} \partial_{31}^{(2)}(v)-\frac{1}{2} z_{32} y \partial_{32} \partial_{21}^{(7)}(v)
\end{aligned}
$$

And

$$
\begin{aligned}
& \left(\delta_{\mathcal{L}_{2} \mathcal{L}_{1}}+\sigma_{1} \circ \delta_{\mathcal{L}_{2} \mathcal{M} \mathcal{M}_{1}}\right)\left(\frac{1}{105} z_{32} y z_{21}^{(2)} x \partial_{21}^{(4)} \partial_{31}(v)-\right. \\
& \left.\frac{1}{7} z_{32} y z_{31} z \partial_{21}^{(6)}(v)\right) \\
& =\sigma_{1}\left(\frac{1}{105} z_{21}^{(2)} x \partial_{21}^{(4)} \partial_{32} \partial_{31}(v)+\right. \\
& \left.\frac{2}{105} z_{21}^{(2)} x \partial_{31} \partial_{21}^{(4)} \partial_{31}(v)\right)+ \\
& \frac{2}{105} z_{21} x \partial_{21}^{(4)} \partial_{31}^{(2)}(v)-\frac{1}{7} z_{32} y \partial_{21}^{(4)} \partial_{31}(v)- \\
& \sigma_{1}\left(z_{32}^{(2)} y \partial_{21}^{(7)}(v)\right)+\frac{1}{7} z_{21} x \partial_{21}^{(6)} \partial_{32}^{(2)}(v)+ \\
& \frac{1}{7} z_{21} x \partial_{21}^{(5)} \partial_{32} \partial_{31}(v)+\frac{1}{7} z_{21} x \partial_{21}^{(4)} \partial_{31}^{(2)}(v)+ \\
& \frac{1}{7} z_{32} y \partial_{31} \partial_{21}^{(6)}(v) \\
& =\frac{1}{42} z_{21} x \partial_{21}^{(5)} \partial_{32} \partial_{31}(v)+\frac{6}{105} z_{21} x \partial_{21}^{(4)} \partial_{31}^{(2)}(v)- \\
& \frac{1}{7} z_{32} y \partial_{21}^{(6)} \partial_{31}(v)-\frac{1}{2} z_{32} y \partial_{32} \partial_{21}^{(7)}(v)+ \\
& \frac{1}{7} z_{21} x \partial_{21}^{(6)} \partial_{32}^{(2)}(v)+\frac{1}{7} z_{21} x \partial_{21}^{(5)} \partial_{32} \partial_{31}(v)+ \\
& \frac{1}{7} z_{21} x \partial_{21}^{(4)} \partial_{31}^{(2)}(v)+\frac{1}{7} z_{32} y \partial_{31} \partial_{21}^{(6)}(v) \\
& =\frac{1}{6} z_{21} x \partial_{21}^{(5)} \partial_{32} \partial_{31}(v)+\frac{1}{5} z_{21} x \partial_{21}^{(4)} \partial_{31}^{(2)}(v)- \\
& \frac{1}{2} z_{32} y \partial_{32} \partial_{21}^{(7)}(v)+\frac{1}{7} z_{21} x \partial_{21}^{(6)} \partial_{32}^{(2)}(v)
\end{aligned}
$$

\section{Proposition 3.2:}

We have exactness at $\mathcal{L}_{i}, i=1,2,3$.

Proof: see (4), (5) and (8).

Now by employ $\sigma_{2}$ we can also acquaint $\partial_{3}: \mathcal{L}_{3} \longrightarrow \mathcal{L}_{2}$ by $\partial_{3}=\delta_{\mathcal{L}_{3} \mathcal{L}_{2}}+\sigma_{2} \circ \delta_{\mathcal{L}_{3} \mathcal{M}_{2}}$

\section{Proposition 3.3:}

The composition $\partial_{1} \circ \partial_{2}$ equal to zero.

Proof: The oneself track employ in proposition 2.

We requirement to acquaint $\sigma_{3}: \mathcal{M}_{3} \longrightarrow \mathcal{L}_{3}$ which implement

$\delta_{\mathcal{M}_{3} \mathcal{L}_{2}}+\sigma_{2} \circ \delta_{\mathcal{M}_{3} \mathcal{M}_{2}}=\left(\delta_{\mathcal{L}_{3} \mathcal{L}_{2}}+\sigma_{2} \circ \delta_{\mathcal{L}_{3} \mathcal{M}_{2}}\right) \circ \sigma_{3}$

As pursue:

- $Z_{21} x Z_{21} x Z_{21} x(v) \mapsto 0$;

where $v \in \mathcal{D}_{11} \otimes \mathcal{D}_{4} \otimes \mathcal{D}_{3}$

- $Z_{21}^{(2)} x Z_{21} x Z_{21} x(v) \mapsto 0$;

where $v \in \mathcal{D}_{12} \otimes \mathcal{D}_{3} \otimes \mathcal{D}_{3}$

- $Z_{21} x Z_{21}^{(2)} x Z_{21} x(v) \mapsto 0$;

where $v \in \mathcal{D}_{12} \otimes \mathcal{D}_{3} \otimes \mathcal{D}_{3}$

- $Z_{21} x Z_{21} x Z_{21}^{(2)} x(v) \mapsto 0$;

where $v \in \mathcal{D}_{12} \otimes \mathcal{D}_{3} \otimes \mathcal{D}_{3}$

- $Z_{21}^{(3)} x Z_{21} x Z_{21} x(v) \mapsto 0$;

where $v \in \mathcal{D}_{13} \otimes \mathcal{D}_{2} \otimes \mathcal{D}_{3}$ 
- $Z_{21} x Z_{21}^{(3)} x Z_{21} x(v) \mapsto 0$; where $v \in \mathcal{D}_{13} \otimes \mathcal{D}_{2} \otimes \mathcal{D}_{3}$

- $Z_{21} x Z_{21} x Z_{21}^{(3)} x(v) \mapsto 0$; where $v \in \mathcal{D}_{13} \otimes \mathcal{D}_{2} \otimes \mathcal{D}_{3}$ - $Z_{21}^{(2)} x Z_{21}^{(2)} x Z_{21} x(v) \mapsto 0$; where $v \in \mathcal{D}_{13} \otimes \mathcal{D}_{2} \otimes \mathcal{D}_{3}$ - $Z_{21}^{(2)} x Z_{21} x Z_{21}^{(2)} x(v) \mapsto 0$; where $v \in \mathcal{D}_{13} \otimes \mathcal{D}_{2} \otimes \mathcal{D}_{3}$

- $z_{21} x z_{21}^{(2)} x z_{21}^{(2)} x(v) \mapsto 0$; where $v \in \mathcal{D}_{13} \otimes \mathcal{D}_{2} \otimes \mathcal{D}_{3}$ - $Z_{21}^{(4)} x Z_{21} x Z_{21} x(v) \mapsto 0$; where $v \in \mathcal{D}_{14} \otimes \mathcal{D}_{1} \otimes \mathcal{D}_{3}$ - $Z_{21} x Z_{21}^{(4)} x Z_{21} x(v) \mapsto 0$; where $v \in \mathcal{D}_{14} \otimes \mathcal{D}_{1} \otimes \mathcal{D}_{3}$

- $Z_{21} x Z_{21} x Z_{21}^{(4)} x(v) \mapsto 0$; where $v \in \mathcal{D}_{14} \otimes \mathcal{D}_{1} \otimes \mathcal{D}_{3}$ - $Z_{21}^{(3)} x Z_{21}^{(2)} x Z_{21} x(v) \mapsto 0$; where $v \in \mathcal{D}_{14} \otimes \mathcal{D}_{1} \otimes \mathcal{D}_{3}$ - $Z_{21}^{(3)} x Z_{21} x Z_{21}^{(2)} x(v) \mapsto 0$; where $v \in \mathcal{D}_{14} \otimes \mathcal{D}_{1} \otimes \mathcal{D}_{3}$ - $z_{21}^{(2)} x z_{21}^{(3)} x z_{21} x(v) \mapsto 0$; where $v \in \mathcal{D}_{14} \otimes \mathcal{D}_{1} \otimes \mathcal{D}_{3}$ - $Z_{21}^{(2)} x Z_{21} x Z_{21}^{(3)} x(v) \mapsto 0$; where $v \in \mathcal{D}_{14} \otimes \mathcal{D}_{1} \otimes \mathcal{D}_{3}$ - $Z_{21} x Z_{21}^{(2)} x Z_{21}^{(3)} x(v) \mapsto 0$; where $v \in \mathcal{D}_{14} \otimes \mathcal{D}_{1} \otimes \mathcal{D}_{3}$ - $Z_{21} x Z_{21}^{(3)} x Z_{21}^{(2)} x(v) \mapsto 0$; where $v \in \mathcal{D}_{14} \otimes \mathcal{D}_{1} \otimes \mathcal{D}_{3}$ - $Z_{21}^{(2)} x Z_{21}^{(2)} x z_{21}^{(2)} x(v) \mapsto 0$; where $v \in \mathcal{D}_{14} \otimes \mathcal{D}_{1} \otimes \mathcal{D}_{3}$ - $Z_{21}^{(5)} x z_{21} x Z_{21} x(v) \mapsto 0$; where $v \in \mathcal{D}_{15} \otimes \mathcal{D}_{0} \otimes \mathcal{D}_{3}$ - $Z_{21} x Z_{21}^{(5)} x Z_{21} x(v) \mapsto 0$; where $v \in \mathcal{D}_{15} \otimes \mathcal{D}_{0} \otimes \mathcal{D}_{3}$ - $Z_{21} x Z_{21} x Z_{21}^{(5)} x(v) \mapsto 0$; where $v \in \mathcal{D}_{15} \otimes \mathcal{D}_{0} \otimes \mathcal{D}_{3}$ - $Z_{21}^{(2)} x Z_{21}^{(2)} x Z_{21}^{(3)} x(v) \mapsto 0$; where $v \in \mathcal{D}_{15} \otimes \mathcal{D}_{0} \otimes \mathcal{D}_{3}$ - $Z_{21}^{(2)} x Z_{21}^{(3)} x Z_{21}^{(2)} x(v) \mapsto 0$; where $v \in \mathcal{D}_{15} \otimes \mathcal{D}_{0} \otimes \mathcal{D}_{3}$

- $z_{21}^{(3)} x z_{21}^{(2)} x z_{21}^{(2)} x(v) \mapsto 0$; where $v \in \mathcal{D}_{15} \otimes \mathcal{D}_{0} \otimes \mathcal{D}_{3}$ - $Z_{21}^{(3)} x z_{21}^{(3)} x z_{21} x(v) \mapsto 0$; where $v \in \mathcal{D}_{15} \otimes \mathcal{D}_{0} \otimes \mathcal{D}_{3}$ - $z_{21}^{(3)} x z_{21} x z_{21}^{(3)} x(v) \mapsto 0$; where $v \in \mathcal{D}_{15} \otimes \mathcal{D}_{0} \otimes \mathcal{D}_{3}$ - $Z_{21} x z_{21}^{(3)} x z_{21}^{(3)} x(v) \mapsto 0$; where $v \in \mathcal{D}_{15} \otimes \mathcal{D}_{0} \otimes \mathcal{D}_{3}$ - $Z_{21}^{(2)} x Z_{21}^{(4)} x z_{21} x(v) \mapsto 0$; where $v \in \mathcal{D}_{15} \otimes \mathcal{D}_{0} \otimes \mathcal{D}_{3}$ - $Z_{21}^{(2)} x Z_{21} x Z_{21}^{(4)} x(v) \mapsto 0$; where $v \in \mathcal{D}_{15} \otimes \mathcal{D}_{0} \otimes \mathcal{D}_{3}$ - $Z_{21} x Z_{21}^{(2)} x Z_{21}^{(4)} x(v) \mapsto 0$; where $v \in \mathcal{D}_{15} \otimes \mathcal{D}_{0} \otimes \mathcal{D}_{3}$ - $Z_{21}^{(4)} x Z_{21}^{(2)} x Z_{21} x(v) \mapsto 0$; where $v \in \mathcal{D}_{15} \otimes \mathcal{D}_{0} \otimes \mathcal{D}_{3}$ - $Z_{21}^{(4)} x Z_{21} x Z_{21}^{(2)} x(v) \mapsto 0$; where $v \in \mathcal{D}_{15} \otimes \mathcal{D}_{0} \otimes \mathcal{D}_{3}$ - $Z_{21} x z_{21}^{(4)} x z_{21}^{(2)} x(v) \mapsto 0$; where $v \in \mathcal{D}_{15} \otimes \mathcal{D}_{0} \otimes \mathcal{D}_{3}$

- $Z_{32} y Z_{21}^{(2)} x Z_{21} x(v) \mapsto 0$; where $v \in \mathcal{D}_{11} \otimes \mathcal{D}_{5} \otimes \mathcal{D}_{2}$ - $z_{32} y z_{21}^{(3)} x z_{21} x(v) \mapsto 0$; where $v \in \mathcal{D}_{12} \otimes \mathcal{D}_{4} \otimes \mathcal{D}_{2}$ - $Z_{32} y z_{21}^{(2)} x z_{21}^{(2)} x(v) \mapsto 0$; where $v \in \mathcal{D}_{12} \otimes \mathcal{D}_{4} \otimes \mathcal{D}_{2}$

- $Z_{32} y z_{21}^{(4)} x Z_{21} x(v) \mapsto 0$; where $v \in \mathcal{D}_{13} \otimes \mathcal{D}_{3} \otimes \mathcal{D}_{2}$ - $z_{32} y z_{21}^{(2)} x z_{21}^{(3)} x(v) \mapsto 0$; where $v \in \mathcal{D}_{13} \otimes \mathcal{D}_{3} \otimes \mathcal{D}_{2}$ - $z_{32} y z_{21}^{(3)} x z_{21}^{(2)} x(v) \mapsto 0$; where $v \in \mathcal{D}_{13} \otimes \mathcal{D}_{3} \otimes \mathcal{D}_{2}$

- $Z_{32} y z_{21}^{(5)} x z_{21} x(v) \mapsto 0$; where $v \in \mathcal{D}_{14} \otimes \mathcal{D}_{2} \otimes \mathcal{D}_{2}$ - $Z_{32} y Z_{21}^{(4)} x z_{21}^{(2)} x(v) \mapsto 0$; where $v \in \mathcal{D}_{14} \otimes \mathcal{D}_{2} \otimes \mathcal{D}_{2}$ - $z_{32} y z_{21}^{(2)} x z_{21}^{(4)} x(v) \mapsto 0$; where $v \in \mathcal{D}_{14} \otimes \mathcal{D}_{2} \otimes \mathcal{D}_{2}$ - $z_{32} y z_{21}^{(3)} x z_{21}^{(3)} x(v) \mapsto 0$; where $v \in \mathcal{D}_{14} \otimes \mathcal{D}_{2} \otimes \mathcal{D}_{2}$ - $z_{32} y z_{21}^{(6)} x z_{21} x(v) \mapsto 0$; where $v \in \mathcal{D}_{15} \otimes \mathcal{D}_{1} \otimes \mathcal{D}_{2}$ - $z_{32} y Z_{21}^{(5)} x z_{21}^{(2)} x(v) \mapsto 0$; where $v \in \mathcal{D}_{15} \otimes \mathcal{D}_{1} \otimes \mathcal{D}_{2}$ - $Z_{32} y Z_{21}^{(2)} x Z_{21}^{(5)} x(v) \mapsto 0$; where $v \in \mathcal{D}_{15} \otimes \mathcal{D}_{1} \otimes \mathcal{D}_{2}$ - $z_{32} y z_{21}^{(4)} x z_{21}^{(3)} x(v) \mapsto 0$; where $v \in \mathcal{D}_{15} \otimes \mathcal{D}_{1} \otimes \mathcal{D}_{2}$ - $z_{32} y z_{21}^{(3)} x z_{21}^{(4)} x(v) \mapsto 0$; where $v \in \mathcal{D}_{15} \otimes \mathcal{D}_{1} \otimes \mathcal{D}_{2}$

- $z_{32} y z_{21}^{(7)} x z_{21} x(v) \mapsto 0$; where $v \in \mathcal{D}_{16} \otimes \mathcal{D}_{0} \otimes \mathcal{D}_{2}$ - $z_{32} y z_{21}^{(6)} x z_{21}^{(2)} x(v) \mapsto 0$; where $v \in \mathcal{D}_{16} \otimes \mathcal{D}_{0} \otimes \mathcal{D}_{2}$ - $z_{32} y z_{21}^{(2)} x z_{21}^{(6)} x(v) \mapsto 0$; where $v \in \mathcal{D}_{16} \otimes \mathcal{D}_{0} \otimes \mathcal{D}_{2}$ - $Z_{32} y z_{21}^{(5)} x Z_{21}^{(3)} x(v) \mapsto 0$; where $v \in \mathcal{D}_{16} \otimes \mathcal{D}_{0} \otimes \mathcal{D}_{2}$ 
- $Z_{32} y Z_{21}^{(3)} x Z_{21}^{(5)} x(v) \mapsto 0$; where $v \in \mathcal{D}_{16} \otimes \mathcal{D}_{0} \otimes \mathcal{D}_{2}$

- $Z_{32} y Z_{21}^{(4)} x Z_{21}^{(4)} x(v) \mapsto 0$; where $v \in \mathcal{D}_{16} \otimes \mathcal{D}_{0} \otimes \mathcal{D}_{2}$

- $z_{32} y z_{32} y z_{21}^{(3)} x(v)$ $\mapsto-\frac{1}{3} z_{32} y z_{31} z z_{21} x \partial_{21}(v)$; where $v \in \mathcal{D}_{11} \otimes \mathcal{D}_{6} \otimes \mathcal{D}_{1}$

- $z_{32} y z_{32} y z_{21}^{(4)} x(v)$ $\mapsto-\frac{1}{6} Z_{32} y Z_{31} z Z_{21} x \partial_{21}^{(2)}(v)$; where $v \in \mathcal{D}_{12} \otimes \mathcal{D}_{5} \otimes \mathcal{D}_{1}$

- $z_{32} y z_{32} y z_{21}^{(5)} x(v)$ $\mapsto-\frac{1}{10} z_{32} y Z_{31} z Z_{21} x \partial_{21}^{(3)}(v)$; where $v \in \mathcal{D}_{13} \otimes \mathcal{D}_{4} \otimes \mathcal{D}_{1}$

- $z_{32} y z_{32} y z_{21}^{(6)} x(v)$ $\mapsto-\frac{1}{15} Z_{32} y Z_{31} z Z_{21} x \partial_{21}^{(4)}(v)$; where $v \in \mathcal{D}_{14} \otimes \mathcal{D}_{3} \otimes \mathcal{D}_{1}$

- $z_{32} y z_{32} y z_{21}^{(7)} x(v)$ $\mapsto-\frac{1}{21} Z_{32} y Z_{31} z Z_{21} x \partial_{21}^{(5)}(v)$; where $v \in \mathcal{D}_{15} \otimes \mathcal{D}_{2} \otimes \mathcal{D}_{1}$

- $z_{32} y z_{32} y z_{21}^{(8)} x(v)$ $\mapsto-\frac{1}{28} z_{32} y z_{31} z Z_{21} x \partial_{21}^{(6)}(v)$; where $v \in \mathcal{D}_{16} \otimes \mathcal{D}_{1} \otimes \mathcal{D}_{1}$

- $z_{32} y z_{32} y z_{21}^{(9)} x(v)$ $\mapsto-\frac{1}{36} Z_{32} y Z_{31} z Z_{21} x \partial_{21}^{(7)}(v)$; where $v \in \mathcal{D}_{17} \otimes \mathcal{D}_{0} \otimes \mathcal{D}_{1}$ - $z_{32}^{(2)} y z_{21}^{(3)} x z_{21} x(v) \mapsto 0$; where $v \in \mathcal{D}_{12} \otimes \mathcal{D}_{5} \otimes \mathcal{D}_{1}$ - $z_{32}^{(2)} y z_{21}^{(4)} x z_{21} x(v) \mapsto 0$; where $v \in \mathcal{D}_{13} \otimes \mathcal{D}_{4} \otimes \mathcal{D}_{1}$

- $z_{32}^{(2)} y z_{21}^{(3)} x z_{21}^{(2)} x(v) \mapsto 0$; where $v \in \mathcal{D}_{13} \otimes \mathcal{D}_{4} \otimes \mathcal{D}_{1}$ - $Z_{32}^{(2)} y Z_{21}^{(5)} x Z_{21} x(v) \mapsto 0$; where $v \in \mathcal{D}_{14} \otimes \mathcal{D}_{3} \otimes \mathcal{D}_{1}$ - $Z_{32}^{(2)} y Z_{21}^{(4)} x Z_{21}^{(2)} x(v) \mapsto 0$; where $v \in \mathcal{D}_{14} \otimes \mathcal{D}_{3} \otimes \mathcal{D}_{1}$ - $z_{32}^{(2)} y z_{21}^{(3)} x z_{21}^{(3)} x(v) \mapsto 0$; where $v \in \mathcal{D}_{14} \otimes \mathcal{D}_{3} \otimes \mathcal{D}_{1}$ - $z_{32}^{(2)} y z_{21}^{(6)} x z_{21} x(v) \mapsto 0$; where $v \in \mathcal{D}_{15} \otimes \mathcal{D}_{2} \otimes \mathcal{D}_{1}$ - $z_{32}^{(2)} y Z_{21}^{(5)} x z_{21}^{(2)} x(v) \mapsto 0$; where $v \in \mathcal{D}_{15} \otimes \mathcal{D}_{2} \otimes \mathcal{D}_{1}$ - $z_{32}^{(2)} y z_{21}^{(4)} x z_{21}^{(3)} x(v) \mapsto 0$; where $v \in \mathcal{D}_{15} \otimes \mathcal{D}_{2} \otimes \mathcal{D}_{1}$ - $z_{32}^{(2)} y z_{21}^{(3)} x z_{21}^{(4)} x(v) \mapsto 0$; where $v \in \mathcal{D}_{15} \otimes \mathcal{D}_{2} \otimes \mathcal{D}_{1}$ - $z_{32}^{(2)} y z_{21}^{(7)} x z_{21} x(v) \mapsto 0$; where $v \in \mathcal{D}_{16} \otimes \mathcal{D}_{1} \otimes \mathcal{D}_{1}$
- $Z_{32}^{(2)} y Z_{21}^{(6)} x Z_{21}^{(2)} x(v) \mapsto 0$; where $v \in \mathcal{D}_{16} \otimes \mathcal{D}_{1} \otimes \mathcal{D}_{1}$ - $z_{32}^{(2)} y Z_{21}^{(5)} x z_{21}^{(3)} x(v) \mapsto 0$; where $v \in \mathcal{D}_{16} \otimes \mathcal{D}_{1} \otimes \mathcal{D}_{1}$

- $z_{32}^{(2)} y Z_{21}^{(4)} x z_{21}^{(4)} x(v) \mapsto 0$; where $v \in \mathcal{D}_{16} \otimes \mathcal{D}_{1} \otimes \mathcal{D}_{1}$ - $Z_{32}^{(2)} y Z_{21}^{(3)} x z_{21}^{(5)} x(v) \mapsto 0$; where $v \in \mathcal{D}_{16} \otimes \mathcal{D}_{1} \otimes \mathcal{D}_{1}$

- $z_{32}^{(2)} y z_{21}^{(8)} x z_{21} x(v) \mapsto 0$; where $v \in \mathcal{D}_{17} \otimes \mathcal{D}_{0} \otimes \mathcal{D}_{1}$ - $z_{32}^{(2)} y z_{21}^{(7)} x z_{21}^{(2)} x(v) \mapsto 0$; where $v \in \mathcal{D}_{17} \otimes \mathcal{D}_{0} \otimes \mathcal{D}_{1}$ - $Z_{32}^{(2)} y Z_{21}^{(6)} x Z_{21}^{(3)} x(v) \mapsto 0$; where $v \in \mathcal{D}_{17} \otimes \mathcal{D}_{0} \otimes \mathcal{D}_{1}$ - $Z_{32}^{(2)} y Z_{21}^{(5)} x Z_{21}^{(4)} x(v) \mapsto 0$; where $v \in \mathcal{D}_{17} \otimes \mathcal{D}_{0} \otimes \mathcal{D}_{1}$ - $z_{32}^{(2)} y z_{21}^{(4)} x z_{21}^{(5)} x(v) \mapsto 0$; where $v \in \mathcal{D}_{17} \otimes \mathcal{D}_{0} \otimes \mathcal{D}_{1}$ - $Z_{32}^{(2)} y Z_{21}^{(3)} x Z_{21}^{(6)} x(v) \mapsto 0$; where $v \in \mathcal{D}_{17} \otimes \mathcal{D}_{0} \otimes \mathcal{D}_{1}$

- $Z_{32} y Z_{32} y Z_{32} y(v) \mapsto 0$; where $v \in \mathcal{D}_{8} \otimes \mathcal{D}_{10} \otimes \mathcal{D}_{0}$ - $z_{32}^{(2)} y z_{32} y z_{21}^{(4)} x(v)$ $\mapsto \frac{1}{6} z_{32} y z_{31} z z_{21} x \partial_{21} \partial_{31}(v)-$ $\frac{1}{4} Z_{32} y Z_{31} z Z_{21} x \partial_{21}^{(2)} \partial_{32}(v)$; where $v \in \mathcal{D}_{12} \otimes \mathcal{D}_{6} \otimes \mathcal{D}_{0}$

- $z_{32}^{(2)} y z_{32} y z_{21}^{(5)} x(v)$ $\mapsto \frac{1}{12} z_{32} y z_{31} z z_{21} x \partial_{21}^{(2)} \partial_{31}(v)-$ $\frac{7}{60} z_{32} y z_{31} z z_{21} x \partial_{21}^{(3)} \partial_{32}(v)$; where $v \in \mathcal{D}_{13} \otimes \mathcal{D}_{5} \otimes \mathcal{D}_{0}$

- $z_{32}^{(2)} y z_{32} y z_{21}^{(6)} x(v)$ $\mapsto \frac{1}{20} z_{32} y z_{31} z z_{21} x \partial_{21}^{(3)} \partial_{31}(v)-$ $\frac{1}{15} Z_{32} y Z_{31} z Z_{21} x \partial_{21}^{(4)} \partial_{32}(v)$; where $v \in \mathcal{D}_{14} \otimes \mathcal{D}_{4} \otimes \mathcal{D}_{0}$

- $z_{32}^{(2)} y z_{32} y z_{21}^{(7)} x(v)$ $\mapsto \frac{1}{30} z_{32} y z_{31} z z_{21} x \partial_{21}^{(4)} \partial_{31}(v)-$ $\frac{3}{70} z_{32} y Z_{31} z Z_{21} x \partial_{21}^{(5)} \partial_{32}(v)$; where $v \in \mathcal{D}_{15} \otimes \mathcal{D}_{3} \otimes \mathcal{D}_{0}$

- $z_{32}^{(2)} y z_{32} y z_{21}^{(8)} x(v)$ $\mapsto \frac{1}{42} z_{32} y z_{31} z z_{21} x \partial_{21}^{(5)} \partial_{31}(v)-$ $\frac{5}{168} z_{32} y z_{31} z z_{21} x \partial_{21}^{(6)} \partial_{32}(v)$; where $v \in \mathcal{D}_{16} \otimes \mathcal{D}_{2} \otimes \mathcal{D}_{0}$

- $z_{32}^{(2)} y z_{32} y z_{21}^{(9)} x(v)$ $\mapsto \frac{1}{56} z_{32} y z_{31} z z_{21} x \partial_{21}^{(6)} \partial_{31}(v)+$ $\frac{1}{72} z_{32} y z_{31} z z_{21} x \partial_{21}^{(7)} \partial_{32}(v)$; where $v \in \mathcal{D}_{17} \otimes \mathcal{D}_{1} \otimes \mathcal{D}_{0}$ 
- $z_{32}^{(2)} y z_{32} y z_{21}^{(10)} x(v)$

$\mapsto \frac{1}{72} Z_{32} y Z_{31} z Z_{21} x \partial_{21}^{(7)} \partial_{31}(v) ;$ where $v \in \mathcal{D}_{18} \otimes \mathcal{D}_{0} \otimes \mathcal{D}_{0}$

- $z_{32} y z_{32}^{(2)} y z_{21}^{(4)} x(v)$ $\mapsto-\frac{1}{3} z_{32} y z_{31} z z_{21} x \partial_{21}^{(2)} \partial_{32}(v)$;

where $v \in \mathcal{D}_{12} \otimes \mathcal{D}_{6} \otimes \mathcal{D}_{0}$

- $z_{32} y z_{32}^{(2)} y z_{21}^{(5)} x(v)$ $\mapsto-\frac{1}{6} z_{32} y z_{31} z z_{21} x \partial_{21}^{(3)} \partial_{32}(v)$; where $v \in \mathcal{D}_{13} \otimes \mathcal{D}_{5} \otimes \mathcal{D}_{0}$

- $z_{32} y z_{32}^{(2)} y z_{21}^{(6)} x(v)$ $\mapsto-\frac{1}{10} z_{32} y z_{31} z z_{21} x \partial_{21}^{(4)} \partial_{32}(v)$; where $v \in \mathcal{D}_{14} \otimes \mathcal{D}_{4} \otimes \mathcal{D}_{0}$

- $z_{32} y Z_{32}^{(2)} y Z_{21}^{(7)} x(v)$ $\mapsto-\frac{1}{15} z_{32} y z_{31} z z_{21} x \partial_{21}^{(5)} \partial_{32}(v)$; where $v \in \mathcal{D}_{15} \otimes \mathcal{D}_{3} \otimes \mathcal{D}_{0}$

- $z_{32} y z_{32}^{(2)} y z_{21}^{(8)} x(v)$ $\mapsto-\frac{1}{21} z_{32} y Z_{31} z Z_{21} x \partial_{21}^{(6)} \partial_{32}(v)$; where $v \in \mathcal{D}_{16} \otimes \mathcal{D}_{2} \otimes \mathcal{D}_{0}$

- $Z_{32} y Z_{32}^{(2)} y Z_{21}^{(9)} x(v) \mapsto 0$; where $v \in \mathcal{D}_{17} \otimes \mathcal{D}_{1} \otimes \mathcal{D}_{0}$

- $z_{32} y z_{32}^{(2)} y z_{21}^{(10)} x(v) \mapsto 0$; where $v \in \mathcal{D}_{18} \otimes \mathcal{D}_{0} \otimes \mathcal{D}_{0}$

- $z_{32}^{(3)} y z_{21}^{(4)} x z_{21} x(v)$ $\mapsto-\frac{1}{9} z_{32} y z_{31} z z_{21} x \partial_{21}^{(2)} \partial_{31}(v)-$ $\frac{1}{18} z_{32} y z_{31} z z_{21} x \partial_{21}^{(3)} \partial_{32}(v)$;

where $v \in \mathcal{D}_{13} \otimes \mathcal{D}_{5} \otimes \mathcal{D}_{0}$

- $Z_{32}^{(3)} y Z_{21}^{(5)} x Z_{21} x(v)$ $\mapsto-\frac{1}{18} Z_{32} y Z_{31} z Z_{21} x \partial_{21}^{(3)} \partial_{31}(v)-$ $\frac{1}{45} z_{32} y z_{31} z z_{21} x \partial_{21}^{(4)} \partial_{32}(v)$;

where $v \in \mathcal{D}_{14} \otimes \mathcal{D}_{4} \otimes \mathcal{D}_{0}$

- $z_{32}^{(3)} y z_{21}^{(4)} x z_{21}^{(2)} x(v)$

$\mapsto-\frac{1}{3} z_{32} y z_{31} z z_{21} x \partial_{21}^{(3)} \partial_{31}(v)-$ $\frac{1}{6} z_{32} y z_{31} z z_{21} x \partial_{21}^{(4)} \partial_{32}(v)$; where $v \in \mathcal{D}_{14} \otimes \mathcal{D}_{4} \otimes \mathcal{D}_{0}$

- $z_{32}^{(3)} y z_{21}^{(6)} x z_{21} x(v)$ $\mapsto-\frac{1}{30} Z_{32} y Z_{31} z Z_{21} x \partial_{21}^{(4)} \partial_{31}(v)-$ $\frac{1}{90} z_{32} y z_{31} z z_{21} x \partial_{21}^{(5)} \partial_{32}(v)$;

where $v \in \mathcal{D}_{15} \otimes \mathcal{D}_{3} \otimes \mathcal{D}_{0}$

- $z_{32}^{(3)} y Z_{21}^{(5)} x z_{21}^{(2)} x(v)$ $\mapsto-\frac{2}{9} z_{32} y z_{31} z z_{21} x \partial_{21}^{(4)} \partial_{31}(v)-$ $\frac{4}{45} z_{32} y z_{31} z z_{21} x \partial_{21}^{(5)} \partial_{32}(v)$;

where $v \in \mathcal{D}_{15} \otimes \mathcal{D}_{3} \otimes \mathcal{D}_{0}$

- $z_{32}^{(3)} y z_{21}^{(4)} x z_{21}^{(3)} x(v)$ $\mapsto-\frac{2}{3} z_{32} y Z_{31} z Z_{21} x \partial_{21}^{(4)} \partial_{31}(v)-$ $\frac{1}{3} Z_{32} y Z_{31} z Z_{21} x \partial_{21}^{(5)} \partial_{32}(v) ;$

where $v \in \mathcal{D}_{15} \otimes \mathcal{D}_{3} \otimes \mathcal{D}_{0}$

- $z_{32}^{(3)} y z_{21}^{(7)} x z_{21} x(v)$

$\mapsto-\frac{1}{45} z_{32} y z_{31} z z_{21} x \partial_{21}^{(5)} \partial_{31}(v)-$ $\frac{2}{315} z_{32} y z_{31} z z_{21} x \partial_{21}^{(6)} \partial_{32}(v)$;

where $v \in \mathcal{D}_{16} \otimes \mathcal{D}_{2} \otimes \mathcal{D}_{0}$

- $z_{32}^{(3)} y z_{21}^{(6)} x z_{21}^{(2)} x(v)$

$\mapsto-\frac{1}{6} Z_{32} y z_{31} z Z_{21} x \partial_{21}^{(5)} \partial_{31}(v)-$

$\frac{1}{18} z_{32} y z_{31} z z_{21} x \partial_{21}^{(6)} \partial_{32}(v)$;

where $v \in \mathcal{D}_{16} \otimes \mathcal{D}_{2} \otimes \mathcal{D}_{0}$

- $z_{32}^{(3)} y z_{21}^{(5)} x z_{21}^{(3)} x(v)$

$\mapsto-\frac{5}{9} Z_{32} y Z_{31} z Z_{21} x \partial_{21}^{(5)} \partial_{31}(v)-$

$\frac{2}{9} Z_{32} y Z_{31} z Z_{21} x \partial_{21}^{(6)} \partial_{32}(v)$;

where $v \in \mathcal{D}_{16} \otimes \mathcal{D}_{2} \otimes \mathcal{D}_{0}$

- $z_{32}^{(3)} y z_{21}^{(4)} x z_{21}^{(4)} x(v)$

$\mapsto-\frac{10}{9} z_{32} y z_{31} z z_{21} x \partial_{21}^{(5)} \partial_{31}(v)-$

$\frac{5}{9} z_{32} y Z_{31} z Z_{21} x \partial_{21}^{(6)} \partial_{32}(v)$;

where $v \in \mathcal{D}_{16} \otimes \mathcal{D}_{2} \otimes \mathcal{D}_{0}$

- $z_{32}^{(3)} y z_{21}^{(8)} x z_{21} x(v)$

$\mapsto-\frac{1}{63} z_{32} y z_{31} z z_{21} x \partial_{21}^{(6)} \partial_{31}(v)-$

$\frac{1}{9} z_{32} y z_{31} z Z_{21} x \partial_{21}^{(7)} \partial_{32}(v)$;

where $v \in \mathcal{D}_{17} \otimes \mathcal{D}_{1} \otimes \mathcal{D}_{0}$

- $z_{32}^{(3)} y z_{21}^{(7)} x z_{21}^{(2)} x(v)$

$\mapsto-\frac{2}{15} z_{32} y z_{31} z z_{21} x \partial_{21}^{(6)} \partial_{31}(v)-$

$\frac{7}{15} z_{32} y z_{31} z z_{21} x \partial_{21}^{(7)} \partial_{32}(v)$;

where $v \in \mathcal{D}_{17} \otimes \mathcal{D}_{1} \otimes \mathcal{D}_{0}$

- $z_{32}^{(3)} y z_{21}^{(6)} x z_{21}^{(3)} x(v)$

$\mapsto-\frac{1}{2} Z_{32} y z_{31} z Z_{21} x \partial_{21}^{(6)} \partial_{31}(v)-$

$\frac{7}{6} z_{32} y Z_{31} z Z_{21} x \partial_{21}^{(7)} \partial_{32}(v)$;

where $v \in \mathcal{D}_{17} \otimes \mathcal{D}_{1} \otimes \mathcal{D}_{0}$

- $z_{32}^{(3)} y z_{21}^{(5)} x z_{21}^{(4)} x(v)$

$\mapsto-\frac{10}{9} z_{32} y z_{31} z z_{21} x \partial_{21}^{(6)} \partial_{31}(v)-$

$\frac{35}{18} z_{32} y z_{31} z z_{21} x \partial_{21}^{(7)} \partial_{32}(v)$;

where $v \in \mathcal{D}_{17} \otimes \mathcal{D}_{1} \otimes \mathcal{D}_{0}$

- $z_{32}^{(3)} y z_{21}^{(4)} x z_{21}^{(5)} x(v)$

$\mapsto-\frac{5}{3} Z_{32} y z_{31} z Z_{21} x \partial_{21}^{(6)} \partial_{31}(v)-$

$\frac{7}{3} z_{32} y z_{31} z z_{21} x \partial_{21}^{(7)} \partial_{32}(v) ;$

where $v \in \mathcal{D}_{17} \otimes \mathcal{D}_{1} \otimes \mathcal{D}_{0}$

- $z_{32}^{(3)} y z_{21}^{(9)} x z_{21} x(v) \mapsto 0$;

where $v \in \mathcal{D}_{18} \otimes \mathcal{D}_{0} \otimes \mathcal{D}_{0}$

- $z_{32}^{(3)} y z_{21}^{(8)} x z_{21}^{(2)} x(v)$

$\mapsto-\frac{1}{9} z_{32} y z_{31} z z_{21} x \partial_{21}^{(7)} \partial_{31}(v)$;

where $v \in \mathcal{D}_{18} \otimes \mathcal{D}_{0} \otimes \mathcal{D}_{0}$ 
- $Z_{32}^{(3)} y Z_{21}^{(7)} x z_{21}^{(3)} x(v)$

$\mapsto-\frac{7}{15} Z_{32} y Z_{31} z Z_{21} x \partial_{21}^{(7)} \partial_{31}(v)$;

where $v \in \mathcal{D}_{18} \otimes \mathcal{D}_{0} \otimes \mathcal{D}_{0}$

- $z_{32}^{(3)} y z_{21}^{(6)} x z_{21}^{(4)} x(v)$

$\mapsto-\frac{7}{6} z_{32} y z_{31} z z_{21} x \partial_{21}^{(7)} \partial_{31}(v)$;

where $v \in \mathcal{D}_{18} \otimes \mathcal{D}_{0} \otimes \mathcal{D}_{0}$

- $z_{32}^{(3)} y z_{21}^{(5)} x z_{21}^{(5)} x(v)$

$\mapsto-\frac{35}{18} z_{32} y z_{31} z z_{21} x \partial_{21}^{(7)} \partial_{31}(v)$

where $v \in \mathcal{D}_{18} \otimes \mathcal{D}_{0} \otimes \mathcal{D}_{0}$

- $z_{32}^{(3)} y z_{21}^{(4)} x z_{21}^{(6)} x(v)$

$\mapsto-\frac{7}{3} z_{32} y z_{31} z z_{21} x \partial_{21}^{(7)} \partial_{31}(v)$;

where $v \in \mathcal{D}_{18} \otimes \mathcal{D}_{0} \otimes \mathcal{D}_{0}$

- $z_{32} y Z_{31} z Z_{21}^{(2)} x(v)$

$\mapsto \frac{1}{3} Z_{32} y z_{31} z Z_{21} x \partial_{21}(v)$;

where $v \in \mathcal{D}_{11} \otimes \mathcal{D}_{6} \otimes \mathcal{D}_{1}$

- $z_{32} y z_{31} z z_{21}^{(3)} x(v)$

$\mapsto \frac{1}{6} Z_{32} y Z_{31} z Z_{21} x \partial_{21}^{(2)}(v)$;

where $v \in \mathcal{D}_{12} \otimes \mathcal{D}_{5} \otimes \mathcal{D}_{1}$

- $z_{32} y z_{31} z z_{21}^{(4)} x(v)$

$\mapsto \frac{1}{10} z_{32} y z_{31} z z_{21} x \partial_{21}^{(3)}(v)$;

where $v \in \mathcal{D}_{13} \otimes \mathcal{D}_{4} \otimes \mathcal{D}_{1}$

- $z_{32} y z_{31} z z_{21}^{(5)} x(v)$

$\mapsto \frac{1}{15} z_{32} y z_{31} z z_{21} x \partial_{21}^{(4)}(v) ;$

where $v \in \mathcal{D}_{14} \otimes \mathcal{D}_{3} \otimes \mathcal{D}_{1}$

- $z_{32} y z_{31} z z_{21}^{(6)} x(v)$

$\mapsto \frac{1}{21} Z_{32} y Z_{31} z Z_{21} x \partial_{21}^{(5)}(v)$;

where $v \in \mathcal{D}_{15} \otimes \mathcal{D}_{2} \otimes \mathcal{D}_{1}$

- $z_{32} y z_{31} z z_{21}^{(7)} x(v)$

$\mapsto \frac{1}{28} z_{32} y z_{31} z z_{21} x \partial_{21}^{(6)}(v)$;

where $v \in \mathcal{D}_{16} \otimes \mathcal{D}_{1} \otimes \mathcal{D}_{1}$

- $z_{32} y z_{31} z z_{21}^{(8)} x(v)$

$\mapsto \frac{1}{36} Z_{32} y Z_{31} z Z_{21} x \partial_{21}^{(7)}(v)$;

where $v \in \mathcal{D}_{17} \otimes \mathcal{D}_{0} \otimes \mathcal{D}_{1}$

- $z_{32} y z_{32} y z_{31} z(v) \mapsto 0$;

where $v \in \mathcal{D}_{9} \otimes \mathcal{D}_{9} \otimes \mathcal{D}_{0}$

- $z_{32}^{(2)} y z_{31} z z_{21}^{(2)} x(v)$

$\mapsto \frac{1}{3} z_{32} y z_{31} z z_{21} x \partial_{31}(v)$;

where $v \in \mathcal{D}_{11} \otimes \mathcal{D}_{7} \otimes \mathcal{D}_{0}$

- $z_{32}^{(2)} y z_{31} z z_{21}^{(3)} x(v)$

$\mapsto \frac{1}{6} z_{32} y z_{31} z z_{21} x \partial_{21} \partial_{31}(v)-$

$\frac{1}{12} z_{32} y z_{31} z z_{21} x \partial_{21}^{(2)} \partial_{32}(v)$;

where $v \in \mathcal{D}_{12} \otimes \mathcal{D}_{6} \otimes \mathcal{D}_{0}$

- $z_{32}^{(2)} y z_{31} z z_{21}^{(4)} x(v)$

$\mapsto \frac{1}{9} Z_{32} y Z_{31} z Z_{21} x \partial_{21}^{(2)} \partial_{31}(v)-$

$\frac{7}{90} z_{32} y z_{31} z z_{21} x \partial_{21}^{(3)} \partial_{32}(v)$; where $v \in \mathcal{D}_{13} \otimes \mathcal{D}_{5} \otimes \mathcal{D}_{0}$

- $z_{32}^{(2)} y z_{31} z z_{21}^{(5)} x(v)$

$\mapsto \frac{1}{12} z_{32} y z_{31} z z_{21} x \partial_{21}^{(3)} \partial_{31}(v)-$

$\frac{1}{15} z_{32} y z_{31} z z_{21} x \partial_{21}^{(4)} \partial_{32}(v)$;

where $v \in \mathcal{D}_{14} \otimes \mathcal{D}_{4} \otimes \mathcal{D}_{0}$

- $z_{32}^{(2)} y z_{31} z z_{21}^{(6)} x(v)$

$\mapsto \frac{1}{15} z_{32} y z_{31} z z_{21} x \partial_{21}^{(4)} \partial_{31}(v)-$

$\frac{2}{35} z_{32} y z_{31} z z_{21} x \partial_{21}^{(5)} \partial_{32}(v)$;

where $v \in \mathcal{D}_{15} \otimes \mathcal{D}_{3} \otimes \mathcal{D}_{0}$

- $z_{32}^{(2)} y z_{31} z z_{21}^{(7)} x(v)$

$\mapsto \frac{1}{18} z_{32} y z_{31} z z_{21} x \partial_{21}^{(5)} \partial_{31}(v)-$

$\frac{25}{504} z_{32} y z_{31} z z_{21} x \partial_{21}^{(6)} \partial_{32}(v)$;

where $v \in \mathcal{D}_{16} \otimes \mathcal{D}_{2} \otimes \mathcal{D}_{0}$

- $Z_{32}^{(2)} y z_{31} z Z_{21}^{(8)} x(v)$

$\mapsto \frac{1}{21} z_{32} y z_{31} z z_{21} x \partial_{21}^{(6)} \partial_{31}(v)+$

$\frac{1}{36} Z_{32} y Z_{31} z Z_{21} x \partial_{21}^{(7)} \partial_{32}(v)$;

where $v \in \mathcal{D}_{17} \otimes \mathcal{D}_{1} \otimes \mathcal{D}_{0}$

- $z_{32}^{(2)} y z_{31} z z_{21}^{(9)} x(v)$

$\mapsto \frac{1}{24} z_{32} y z_{31} z z_{21} x \partial_{21}^{(7)} \partial_{31}(v)$;

where $v \in \mathcal{D}_{18} \otimes \mathcal{D}_{0} \otimes \mathcal{D}_{0}$

Again we can exhibit that $\sigma_{3}$ which is realized above implement 4 , and we adopt one of them as an example

Where $v \in \mathcal{D}_{12} \otimes \mathcal{D}_{5} \otimes \mathcal{D}_{1}$

$\left(\delta_{\mathcal{M}_{3} \mathcal{L}_{2}}+\sigma_{2} \circ \delta_{\mathcal{M}_{3} \mathcal{M}_{2}}\right)\left(Z_{32} y Z_{31} z Z_{21}^{(3)} x(v)\right)$

$=\sigma_{2}\left(2 z_{32}^{(2)} y z_{21}^{(4)} x \partial_{32}^{(2)}(v)-z_{21} x \partial_{32}^{(2)} z_{21}^{(3)} x(v)+\right.$

$\left.z_{32} y z_{21}^{(4)} x \partial_{32}(v)-z_{32} y z_{32} y \partial_{21}^{(4)}(v)\right)+$

$z_{32} y z_{31} z \partial_{21}^{(3)}(v)$

$=\frac{1}{6} z_{32} y z_{21}^{(2)} x \partial_{21} \partial_{31}(v)+\frac{1}{2} z_{32} y z_{31} z \partial_{21}^{(3)}(v)$

$+\frac{1}{6} z_{32} y z_{21}^{(2)} x \partial_{21}^{(2)} \partial_{32}(v)$

And

$\left(\delta_{\mathcal{L}_{3} \mathcal{L}_{2}}+\sigma_{2} \circ \delta_{\mathcal{L}_{3} \mathcal{M}_{2}}\right)\left(\frac{1}{6} Z_{32} y Z_{31} z Z_{21} x \partial_{21}^{(2)}(v)\right)$

$=\sigma_{2}\left(\frac{1}{6} z_{21} x Z_{21} x \partial_{21}^{(2)} \partial_{32}^{(2)}(v)\right)+$

$\frac{1}{6} z_{32} y z_{21}^{(2)} x \partial_{32} \partial_{21}^{(2)}(v)+$

$\frac{1}{6} z_{32} y z_{21}^{(2)} x \partial_{21} \partial_{31}(v)-$

$\sigma_{2}\left(z_{32} y Z_{32} y \partial_{21}^{(4)}(v)\right)+\frac{1}{2} Z_{32} y Z_{31} z \partial_{21}^{(3)}(v)$

$=\frac{1}{6} z_{32} y z_{21}^{(2)} x \partial_{21}^{(2)} \partial_{32}(v)+$

$\frac{1}{6} z_{32} y Z_{21}^{(2)} x \partial_{21} \partial_{31}(v)+$

$\frac{2}{4} z_{32} y z_{31} z \partial_{21}^{(3)}(v)$

So from all, we have done above we have the complex 


$$
0 \longrightarrow \mathcal{L}_{3} \stackrel{\partial_{3}}{\longrightarrow} \mathcal{L}_{2} \stackrel{\partial_{2}}{\longrightarrow} \mathcal{L}_{1} \stackrel{\partial_{1}}{\longrightarrow} \mathcal{L}_{0}
$$

Where $\partial_{1}$ is the operation of indicated polarization operators, $\partial_{2}$ acquaint as pursue

- $\partial_{2}\left(Z_{21} x(v)\right)=\partial_{21}(v)$;

where $v \in \mathcal{D}_{9} \otimes \mathcal{D}_{6} \otimes \mathcal{D}_{3}$

- $\partial_{2}\left(z_{32} y(v)\right)=\partial_{32}(v)$;

where $v \in \mathcal{D}_{8} \otimes \mathcal{D}_{8} \otimes \mathcal{D}_{2}$

- $\partial_{2}\left(Z_{32} y Z_{21}^{(2)} x(v)\right)=\frac{1}{2} z_{21} x \partial_{21} \partial_{32}(v)+$

$z_{21} x \partial_{31}(v)-z_{32} y \partial_{21}^{(2)}(v)$;

where $v \in \mathcal{D}_{10} \otimes \mathcal{D}_{6} \otimes \mathcal{D}_{2}$

- $\partial_{2}\left(Z_{32} y Z_{31} z(v)\right)=\frac{1}{2} z_{32} y \partial_{32} \partial_{21}(v)+$ $z_{21} x \partial_{32}^{(2)}(v)-z_{32} y \partial_{32}^{(2)}(v)$;

where $v \in \mathcal{D}_{9} \otimes \mathcal{D}_{8} \otimes \mathcal{D}_{1}$

And the map $\partial_{3}$ acquaint as

- $\partial_{2}\left(Z_{32} y Z_{31} z Z_{21} x(v)\right)=Z_{32} y Z_{21}^{(2)} x \partial_{32}(v)+$ $Z_{32} y Z_{31} z \partial_{21}(v) ;$ where $v \in \mathcal{D}_{10} \otimes \mathcal{D}_{7} \otimes \mathcal{D}_{1}$

\section{Proposition 3.4:}

The complex

$\underset{\mathrm{O}}{\longrightarrow} K_{(8,7,3)} \mathcal{L}_{3} \stackrel{\partial_{3}}{\longrightarrow} \mathcal{L}_{2} \stackrel{\partial_{2}}{\longrightarrow} \mathcal{L}_{1} \stackrel{\partial_{1}}{\longrightarrow} \mathcal{L}_{0}$

is exact.

Proof: see (4), (5) and (8).

\section{Acknowledgments}

The authors thank Mustansiriyah University / College of Science / Department of Mathematics and University of Baghdad / College of Education for Pure Science - Ibn Al-Haitham / Department of Mathematics for their supported this work.

\section{Conflicts of Interest: None.}

\section{References:}

1. David AB, Taylor B.D. Homotopies for Resolution of Skew-Hook Shapes. Adv Appl Math.2003; 30(0196-8858):26-43.

2. Haytham RH. The Reduction of Resolution of Weyl Module From Characteristic-Free Resolution in Case $(4,4,3)$. IHJPAS. 2012; 25(3)(2521-3407):341-355.

3. Giandomenico B, David AB. Threading Homology Through Algebra: Selected Patterns. Clorendon Press. Oxford; 2006.

4. Haytham RH, Mays MM. Application of the resolution of the characteristic-free resolution of Weyl module to Lascoux resolution in case $(6,6,3)$. Int J Eng Appl Sci. 2016; 3(23943661):57-63.

5. Najah MM. Resolution of Wely Module in the Case of the Partition (7,6,3). M.Sc. Thesis: College of Science, Al-Mustansiriyah University, Iraq; 2017.

6. Haytham RH, Niran SJ. Application of Weyl Module in the Case of Two Rows. J.Phys.:Conf.Ser. 2018; 1003 (012051)(1742 6588,742 - 6596): $1-15$, doi :10.1088/17426596/1003/1/012051.

7. Haytham RH, Niran SJ. A Complex of Characteristic Zero in the Case of the Partition $(8,7,3)$. Sci. Int. (Lahore). 2018; 30(4)(10135316):639-641.

8. David AB. A Characteristic Free Example of Lascoux Resolution, and Letter Place Methods for Intertwining Numbers. Eur J Comb.2004; 25(0195-6698):1169-1179.

9. Haytham RH. Application of the characteristicfree resolution of Weyl Module to the Lascoux resolution in the case $(3,3,3)$. Ph.D. Thesis: Universita di Roma "Tor Vergata", Italian (Roma); 2006.

10.Haytham RH, Najah MM. Complex of Lascoux in the Case of Partition (7,6,3). Aust J Basic Appl Sci. 2016; 10(18)(1991-8178):89-93.

\section{حول التحلل الحر لمقاس وايل وتحلل المميز الصفري في حالة التجزئة (8,7,3)

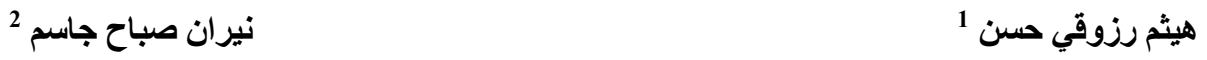

1قسم الرياضيات، كلية العلوم، الجامعة المستتصرية، بغداد، العراق.

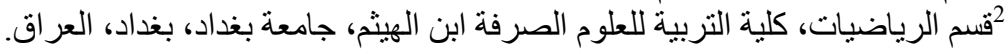

